\title{
Distributed Location Estimation of a Moving Target Characterized by a Spatial Poisson Field
}

\author{
Fathi Masoud \\ West Virginia University
}

Follow this and additional works at: https://researchrepository.wvu.edu/etd

\section{Recommended Citation}

Masoud, Fathi, "Distributed Location Estimation of a Moving Target Characterized by a Spatial Poisson Field" (2013). Graduate Theses, Dissertations, and Problem Reports. 532.

https://researchrepository.wvu.edu/etd/532

This Thesis is protected by copyright and/or related rights. It has been brought to you by the The Research Repository @ WVU with permission from the rights-holder(s). You are free to use this Thesis in any way that is permitted by the copyright and related rights legislation that applies to your use. For other uses you must obtain permission from the rights-holder(s) directly, unless additional rights are indicated by a Creative Commons license in the record and/ or on the work itself. This Thesis has been accepted for inclusion in WVU Graduate Theses, Dissertations, and Problem Reports collection by an authorized administrator of The Research Repository @ WVU. For more information, please contact researchrepository@mail.wvu.edu. 


\title{
Distributed Location Estimation of a Moving Target Characterized by a Spatial Poisson Field
}

\author{
by \\ Fathi Masoud \\ Thesis submitted to the \\ College of Engineering and Mineral Resources \\ at West Virginia University \\ in partial fulfillment of the requirements \\ for the degree of \\ Master of Science \\ in \\ Electrical Engineering \\ Matthew C. Valenti, Ph.D. \\ Daryl S. Reynolds, Ph.D. \\ Natalia A. Schmid, DSc., Chair \\ Lane Department of Computer Science and Electrical Engineering \\ Morgantown, West Virginia \\ 2013
}

Keywords: Maximum Likelihood Estimation, Bisection rule, Secant method, Tracking Simulation, SNR, Sensor Network, Channel Bandwidth 


\author{
Abstract \\ Distributed Location Estimation of a Moving Target Characterized by a Spatial Poisson \\ Field \\ by \\ Fathi Masoud \\ Master of Science in Electrical Engineering \\ West Virginia University \\ Natalia A. Schmid, DSc., Chair
}

Wireless Sensor Networks (WSNs) are traditionally employed to collect spatial and temporal data characterizing various events. These data are then used to solve inference problems such as object detection, counting, classification, estimation and tracking. Distributed solutions provided by WSNs are often cost effective and characterized by high performance indices.

In this work, we model and simulate a distributed sensor network composed of radiation detectors and analyze its ability to make inferences. Radiation detectors are deployed over a known area. A radiological point source is positioned in the interior of the area. Detectors take measurements of the field generated by the point source and transmit them (without any interaction with one another) to a remotely installed super computer (called here Fusion Center) for a joint processing. To minimize consumption of resources such as power in the network and transmission bandwidth, the measurements are locally preprocessed prior to transmission. Our model assumes two Gaussian channels, observation and transmission. The first channel distorts data at the receiver end of each sensor during data acquisition. The second channel distorts data during transmission. Sensor measurements are modeled as an inhomogeneous spatial counting random process (Poisson process). The location of the radiological point source in the area and the strength of the field generated by the substance are unknown parameters. The goal of the FC is to estimate these parameters from the distributed measurements provided by the WSN. To find the distributed estimates, we adopt the Maximum Likelihood approach. This approach requires knowledge of the joint probability density function of the distributed measurements observed by the FC. Since the joint probability density of the data observed at the $\mathrm{FC}$ is nonlinear in unknown parameters, we propose an iterative approach to solve for the maximum likelihood estimates of these parameters. The solution is a combination of the Bisection and Secant approaches adjusted to seek solution in a multidimensional parameter space. The performance of the distributed estimator is measured in terms of the mean square error. It is analyzed with respect to various parameters of the WSN. We vary the following parameters of the network: (1) the number of sensors in the WSN, (2) signal to noise ratio in observation and transmission channels, (3) the strength of the original field, and (4) the number of quantization levels used by a sensor to convert an analog measurement into a digital signal. We also propose a distributed tracking algorithm for monitoring position of the object in real time. 


\section{Acknowledgements}

I owe my deepest gratitude and thanks to my advisor, Dr. Schmid, for giving me the opportunity to work with her. This thesis would not be possible without her guidance and support. Also it is an honor for me to thank Dr.Valenti and Dr. Reynolds for being on my committee. The opportunity to take courses with Dr.Valenti, gave me essential understanding of the subject. Next, I would also like to thank my colleagues and friends for their help, specially M. Alkhweldi for his counsel and useful advices. Finally, I would like to express my gratitude to my family members for their constant support and encouragement. 


\section{Contents}

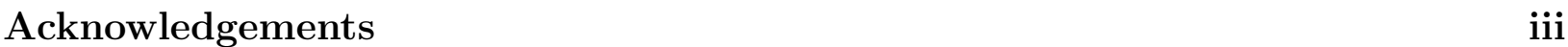

List of Figures $\quad$ vi

1 Introduction $\quad 1$

2 Literature Review $\quad 4$

2.1 Papers related to optimizing WSN parameters under constraints . . . . . . . 4

2.2 Sensor node location estimation . . . . . . . . . . . . . . . 5

2.3 Target localization . . . . . . . . . . . . . . . . . . . 6

2.3.1 Localization in the presence of uncertainties . . . . . . . . 6

2.3.2 Target Location Estimation _. . . . . . . . . . . . . . . . . 6

3 Problem statement and proposed solution $\quad 8$

3.1 Sensor Measurements . . . . . . . . . . . . . . . . . . . . 9 9

3.2 Local Processing for Digital Case . . . . . . . . . . . . . . . . . . . 9

3.3 Problem Statement . . . . . . . . . . . . . . . . . . . . 10

3.4 Proposed Solution . . . . . . . . . . . . . . . . . . . . . . 10

3.5 Maximum Likelihood Solution . . . . . . . . . . . . . . . . . 10

3.5.1 Models for the Analog Case . . . . . . . . . . . . . . . . . 10

3.5.2 Maximum Likelihood Estimation (analog case) . . . . . . . . . . . . . . 11

3.5.3 Models for the Digital Case . . . . . . . . . . . . . . . . . 13

3.5.4 Maximum Likelihood Estimation (digital case) . . . . . . . . . . . . 14

3.6 Iterative solution . . . . . . . . . . . . . . . . . . . . 15

4 Numerical Results $\quad 20$

4.1 Signal to Noise Ratio . . . . . . . . . . . . . . . . . . . . . . . . . 21

4.1 .1 Observation SNR . . . . . . . . . . . . . . . . . . . . 22

4.1 .2 Transmission SNR . . . . . . . . . . . . . . . . . . . . . 23

4.2 Analog Channel . . . . . . . . . . . . . . . . . . 26

4.2.1 Effect of sensors density on the estimation performance . . . . . . . 26

4.2 .2 Initialization of iterative algorithm . . . . . . . . . . . 26

4.2.3 Effect of the strength of the field on the estimation error . . . . . . . 28

4.3 Digital Channel . . . . . . . . . . . . . . . . . . . . 29

4.3.1 Effect of sensor density on the estimation performance: digital case . 29 
4.3.2 Effect of the number of quantization levels $M$ on the estimation performance . . . . . . . . . . . . . . . . 29

4.3.3 Initialization of iterative algorithm: digital case . . . . . . . . . . 31

4.3.4 Effect of the strength of the field on the estimation error: digital case 31

4.4 Effect of Signal-to-Noise Ratio on the estimation performance . . . . . . . . 33

4.5 Probability of Outliers . . . . . . . . . . . . . . . . . . . . . . . . . . . . . . . 35

4.6 Tracking Simulation . . . . . . . . . . . . . . . . . . 36

5 Conclusion, discussion, and future work $\quad 45$

5.1 Summary . . . . . . . . . . . . . . . . . . . . . . 45

5.2 Claimed Novelties . . . . . . . . . . . . . . . . . . . . . . 46

5.3 Future Work . . . . . . . . . . . . . . . . . . . . . . 47

$\begin{array}{ll}\text { References } & 48\end{array}$ 


\section{List of Figures}

1.1 Sensor components. . . . . . . . . . . . . . . . . . . . 1

3.1 A block diagram of the Bisection method. . . . . . . . . . . . . . . 17

3.2 The combination of the two methods, Bisection and Secant. . . . . . . . . 18

4.1 The area $\Omega$, limits of Polar Integration . . . . . . . . . . . . . . . . 23

4.2 A box plot of SE as a function of the number of sensors $K$ placed within the area $\Omega$ for the analog case. $S N R_{0}=27 d B$, and $\sigma_{c}^{2}=0.8 \ldots \ldots \ldots . . . . .27$

4.3 A box plot of $\mathrm{SE}$ as a function of a region, where initial values of estimates are drawn. $S N R_{0}=27 d B$, and $\sigma_{c}^{2}=0.8 \ldots \ldots \ldots \ldots . \ldots . \ldots . \ldots 27$

4.4 SE versus $\Lambda, \sigma_{0}^{2}$ is set to 0.8 , analog case. . . . . . . . . . . . . 28

4.5 A box plot of SE as a function of the number of sensors $K$ placed within the area $\Omega$ for the digital case. $S N R_{0}=27 d B, \sigma_{c}^{2}=0.8$, and $M=16$ levels. . . . . . 30

4.6 A box plot of SE as a function of the number of quantization levels $M$.

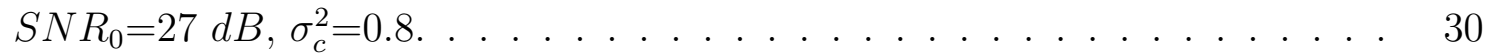

4.7 SE versus Ring Labels, $\Lambda=100, \sigma_{0}^{2}=0.8$, and $M=16$ : digital case. . . . . . . 32

4.8 SE versus $\Lambda, \sigma_{0}^{2}=0.8, K=100$, and $M=16$ : digital case. . . . . . . . . . 32

4.9 SE versus $S N R_{0}, \sigma_{c}^{2}=0.003 \mathrm{~dB}, K=10$, analog case. . . . . . . . . . . 33

$4.10 \mathrm{SE}$ versus $S N R_{0}, S N R_{c}=40 \mathrm{~dB}, M=8, K=10$, digital case. . . . . . . . . 34

$4.11 \mathrm{SE}$ versus $S N R_{c}, S N R_{0}=20 \mathrm{~dB}, K=10$, analog case. . . . . . . . . . . 34

$4.12 \mathrm{SE}$ versus $S N R_{c}, S N R_{0}=20 \mathrm{~dB}, M=8, K=10$, digital case. . . . . . . . . 35

4.13 Probability of outliers, $\Lambda=100, K=20$, and $\sigma_{0}^{2}=0.8$ : analog case. . . . . . . 36

4.14 Probability of outlier, $\Lambda=100, K=20, \sigma_{0}^{2}=0.8$, and $M=16$ : digital case. . . . 36

4.15 Tracking Simulation, $\Lambda=100, S N R_{0}=27 d B, \sigma_{c}^{2}=0.8$, analog case. . . . . . . 38

4.16 Tracking Simulation, $\Lambda=100, S N R_{0}=27 d B, \sigma_{c}^{2}=0.8, M=16$, digital case. . . 38

4.17 Tracking Simulation, $\Lambda=100, S N R_{0}=27 d B, \sigma_{c}^{2}=0.8$, analog case. . . . . . 39

4.18 Tracking Simulation, $\Lambda=100, S N R_{0}=27 d B, \sigma_{c}^{2}=0.8, M=16$, digital case. . . 39

4.19 Tracking Simulation, $\Lambda=100, S N R_{0}=27 d B, \sigma_{c}^{2}=0.8$, analog case. . . . . . 40

4.20 Tracking Simulation, $\Lambda=100, K=400, S N R_{0}=27 d B, \sigma_{c}^{2}=0.8$, analog case. $\quad 40$

4.21 Tracking Simulation, $\Lambda=100, K=100, S N R_{0}=27 d B, \sigma_{c}^{2}=0.8, M=8$, digital case. . . . . . . . . . . . . . . . . . . . 41

4.22 Tracking Simulation, $\Lambda=100, K=100, S N R_{0}=27 d B, \sigma_{c}^{2}=0.8, M=32$, digital case. . . . . . . . . . . . . . . . . . . . 41

4.23 Tracking Simulation, $\Lambda=30, K=100, S N R_{0}=15 d B, \sigma_{c}^{2}=0.8$, analog case. . . 42 
4.24 Tracking Simulation, $\Lambda=30, K=100, S N R_{0}=15 d B, \sigma_{c}^{2}=0.8, M=32$, digital case. . . . . . . . . . . . . . . . . . . 43

4.25 Tracking Simulation at a pair of points on the boundary of the area: $\Lambda=100$, $K=100, S N R_{0}=27 d B, \sigma_{c}^{2}=0.8$, analog case. . . . . . . . . . . . . 44

4.26 Tracking Simulation with initializations at the boundary of the area: $\Lambda=100$, $K=100, S N R_{0}=27 d B, \sigma_{c}^{2}=0.8$, digital case. . . . . . . . . . . . . 44 


\section{Chapter 1}

\section{Introduction}

Wireless Sensor Networks WSNs are data acquiring and managing systems. They consist of multiple wireless sensor nodes. A typical node contains a processing unit, sensing transducer, radio transceiver, and a power supply. A general block diagram is illustrated in Figure 1 [1]. The processing unit controls the node, performs the communication protocols

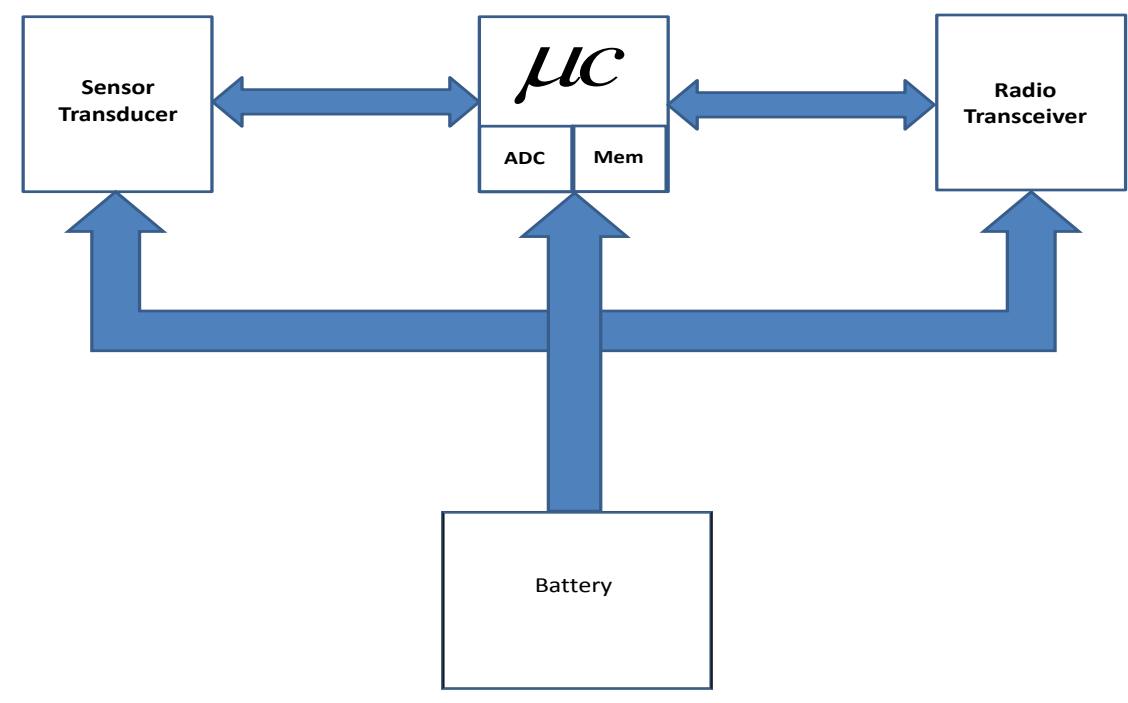

Figure 1.1: Sensor components.

and includes the memory, micro controller and analog to digital converter [2]. The sensor transducer senses the intended environmental phenomena and converts the sensed data into an electrical signal. A battery feeds the whole node. It is an important component of the node, as the lifetime of the nodes depends on it. A radio transceiver links the nodes with 
each other and with the network main operation center.

WSN topology depends on the application, as the topology structure plays a major role in determining the network performance [3]. Sensors can be connected to each other and form mesh network, and could be organized around a central hub (star topology). The topology might be more complex such as the clustered hierarchy networks $[4,5,6,7]$.

WSN is utilized to monitor environmental changes such as temperature, humidity, light, electromagnetic fields, and fumes. It is also used in control applications, machine health monitoring, military and agricultural applications. The collected data are sent over wireless channel to a processing center, which is a super computer (Fusion Center) that jointly processes the data by using a specific algorithm for required applications [8, 9, 10, 11].

WSN facilitates discovering our environment and acquiring data. This technology is improving in parallel with the improving of infrastructure technology like semiconductors, capacity of batteries and memory performance [12]. Limited battery power and limited transmission bandwidth of communication channels are two main constraints that limit performance of WSNs. Many investigators have studied the effect of each constraint $[13,14,15]$ and both constraints [16] on the performance of WSNs. Many applications such as distributed estimation, detection, object counting, and tracking have been analyzed [17].

Distributed detection and localization of objects (substance) are the key applications of WSNs. Object localization is a type of estimation problem, where position of an object is estimated [18]. In addition to location estimation, other parameters such as signal or field parameters can be estimated [19].

In this thesis we develop an object localization approach by using distributed measurements of a WSN of radiation detectors. We assume that a radioactive object (substance) is slowly moving across the area with deployed wireless sensors. The object generates a field modeled as an inhomogeneous spatial Poison process. Radioactive detectors take measurements of this field and transmit them to a Fusion Center (FC) for a joint processing. We assume that both observation and transmission channels distort sensor measurements and model distortions as Gaussian random variables.

We take Maximum Likelihood ML Estimation approach to solve for unknown parameters. This requires forming a joint likelihood function of noisy measurements at FC. The neces- 
sary condition to find a maximizer of the likelihood function result in a set of equations that are highly nonlinear in estimated position. Therefore, we propose an iterative algorithm to solve for the location estimates. The proposed algorithm is a combination of two well known approaches, Secant and Bisection [20, 21, 22].

We analyze performance of the distributed estimator with respect to a number of parameters such as the number of sensors in the WSN, Signal-to-Noise Ratio in observation and transmission channels, number of quantization levels, and other parameters.

Two cases are considered, analog and digital. In the analog case, sensor measurements are sent immediately to the FC over a communication channel, while in the digital case the measurements are digitized by a common quantizer before they are transmitted by the channel. The novelty of our work comes from localizing a target characterized by a random Poisson field. The proposed algorithm is used to solve for the location that maximizes the likelihood estimation function, where two numerical methods are combined, Bisection and Secant, in order to benefit from the speed of Secant method and the reliability of Bisection rule.

The rest of the thesis is organized as follows. Chapter 2 includes a review of relevant works. Chapter 3 presents our problem statement and proposed solution. The joint probability density function $(p d f)$ is derived for both digital and analog cases, and used to form the Maximum Likelihood Estimation functions, which is solved by means of the proposed iterative algorithm. Chapter 4 presents numerical analysis. The graphs are plotted to find the optimal parameters, in order to use these parameters for tracking purposes. Tracking simulations are presented for both digital and analog cases and for different target moving paths. Also, the Signal to Noise Ratio (SNR) is defined for the observation and for the communication channels.

Chapter 5 states the summary, conclusion, and future work. The results show that the used algorithm is efficient, robust and relatively simple. Its simplicity is attributed to a basic way of combining Bisection and Secant methods into a single algorithm. Literature contains few algorithms combining the two methods, but they all have high complexity. 


\section{Chapter 2}

\section{Literature Review}

Since their introduction in 1980s WSNs have become an active research topic. Many new developments have been documented and performance analysis has been performed. All publications on the topic available today can be broadly partitioned into publications providing a general overview of WSNs, publications that describe physical constraints of WSNs, develop new optimized solutions and analyze their performance, and publications devoted to limits (sensing and communications) that can be achieved in practice.

In this chapter we will focus only on a small portion of these publications. We will summarize a number of publications devoted to the problem of distributed target localization in the presence of uncertainties and constraints.

\subsection{Papers related to optimizing WSN parameters un- der constraints}

1. The paper by Ribeiro et al [23] considers a distributed estimation problem. Under the bandwidth constraint of 1 bit per sensor, they found that an optimal quantization step could be applied to get the best variance for a given bandwidth constraint. They concluded that choosing the quantization step equal to the noise variance is sufficient for many practical cases.

2. Junlin [24] introduced MSE function. It is used to estimate the optimal bit rate 
and transmission energy of each user. The MSE is minimized under a given total energy budget. An optimal distributed estimation algorithm for homogeneous sensors and a quasi-optimal distributed algorithm for heterogeneous sensors are proposed and analyzed.

3. In [25] a decentralized estimation scheme, SES, is built by Xiao et al to find an unknown parameter, which is modeled as a discrete random variable. Encoding and decoding of sensor measurements is based on the idea that the length of the digitized sensor measurement is proportional to the logarithm of its local signal to noise ratio (SNR). The compressed data are then combined by the Fusion Center. The main advantages of the scheme are the economic power transmission and the low communication requirements.

\subsection{Sensor node location estimation}

1. Received Signal Strength Indicator (RSSI) is proposed by Al Alawi in [26]. The measurement is used to estimate sensor node location in WSN. Practical data, collected both indoor and outdoor, are used in the experiments. Calibration model is needed to demonstrate RF radio channel, which is included in the estimation procedures. Since the model is built according to RSSI measurements, it is used to estimate the distance between an unknown node location and known anchor location.

2. In [27] Chen introduced a location estimation algorithm. The purpose of this algorithm is to find the position of a target sensor node in WSN at certain point in space and time. The algorithm does not assume any synchronization between the nodes of the network. Instead, it uses a time difference scheme. Time Difference of Arrival (TDOA) location algorithm and Kalman filtering are both adopted. Sensors do not send any RF signal, which apparently reduces the total power consumption of the system. 


\subsection{Target localization}

\subsubsection{Localization in the presence of uncertainties}

1. The authors of [28] propose the ML target localization approach in WSN. The paper assumes an imperfect BSC channel, which links the Fusion Center and the sensors. Sensor measurements are quantized, and the statistics of the imperfect channel as well as decoding scheme characteristics at the receiver are included in the localization algorithm. Simulations are performed to illustrate that the performance under these practical parameters is close to the theoretically derived performance.

2. The paper [29] analyzes the situation of malfunctioning sensors in WSN. Two cases are considered here, the case of independent false sensors and the case of connected false sensors. The sensors send binary data to the Fusion Center. Cramer-Rao Lower Bound and Fisher Information Matrix are utilized in this paper to evaluate the network functionality in the presence of false information (Byzantine Attacks).

\subsubsection{Target Location Estimation}

1. An intensity-based ML target location estimator is introduced in [30]. Ruixin assumes that a signal generated by the target is isotropic, and its intensity attenuates as a function of inverse power $n$ distance from the target. Sensor measurements are quantized and sent to a Fusion Center. The paper presents a derivation of the Carmer-Rao Lower Bound on the variance of parameter estimates. It is also shown through simulations that the results generated by the ML estimator are more precise than those of heuristic weighted average methods. Furthermore, optimal quantization thresholds are calculated to attain a better estimation performance.

2. In [31] an algorithm is presented by Jingjing to estimate location of a target. This algorithm is based on calculating azimuth angle by utilizing Time Delay of Arrival TDOA, which is measured by each sensor in a WSN. Then the reading (arrival delays) of each group of sensors (cluster) is calculated by a cluster head. The angles and the cluster locations are then used to estimate the source location. 
3. The purpose of Sheng in [32] is to reduce the number of sensor nodes and time cost. The idea is suggested by a theory of particle filtering called distributed lightweight particle filter algorithm. Each sensor node performs local particle filtering using local measurements and the measurements of a neighboring sensor. These measurements are compared in order to make the ultimate decision. Some of experiments outcomes are illustrated to evaluate the performance of MLE, Centralized Particle Filter CPF and the proposed Distributed Lightweight Particle Filter DLPF.

4. The authors of [33] introduced a WSN that has a symmetric-tree structure and used the network to perform multi-target tracking. They also analyzed the decay rate of the error detection probability.

5. The paper by Sangeetha [34] uses inverse transformation and Kalman filter to estimate the location of a target. Kalman filter is used to develop the estimation strategy and decrease the effect of noise on the accuracy of results. It is also used for the purpose of estimating signal. Measured Error Covariance and Estimation Error Covariance are utilized to evaluate the performance of the experimental outcomes.

6. The work [35] introduced an algorithm that uses directional sensors rather than omnidirectional for target tracking. This provides sensors with a certain field of view. The solution is to control the direction of sensor coverage to save power and have efficient results. The algorithm can be used in electronic steering and in the mechanical steering. Each sensor is steered only when it detects a target. The sensor tracks the target until it reaches the field of view of another sensor.

7. Mansouri adopts in [36] the idea of choosing a group of sensors rather than the entire sensor network. He assumed that sensor measurements are quantized. One of the goals of the paper is to find an optimal quantization strategy for the purpose of preserving the bandwidth in transmission channel. The proposed algorithm suggests to simultaneously finding the best group of sensors and performing quantization by means of a "multi-objective" function. 


\section{Chapter 3}

\section{Problem statement and proposed solution}

In this chapter, we state the problem of distributed estimation of a parametric stochastic field as a maximum likelihood estimation problem and suggest an iterative solution to it. The solution alternates a bi-section rule and a secant method, which results in an efficient and robust estimation algorithm.

Assume a WSN is composed of a fixed number of sensors. The sensors are randomly distributed over an area $\Omega$ with known locations $\left(x_{i}, y_{i}\right)$ of the $i^{\text {th }}$ sensor $i=1 \ldots K$. Assume further that an object (such as car, truck, military vehicle, etc) or a subject equipped with a radioactive substance is moving along a path in the area of interest. The radioactive substance generates a field measured by the distributed WSN. Each sensor takes a sample $r\left(x_{i}, y_{i}\right)$ of the field and sends it immediately over a communication media to a super computer, called here Fusion Center (FC), for a joint data processing. We consider two cases of data processing and communication channels: analog and digital. In the case of analog channel, sensory data are sent as they are by means of linear or nonlinear modulation [37]. A different processing is performed in the digital case, where a sample is sent by sensor to a local Quantizer, where it is digitized before sending it to the FC. We assume that communication channels are parallel Additive White Gaussian Noise channels (AWGN)[38]. Therefore the Fusion Center receives independent sensory data. The Fusion Center combines noisy sensory data and estimates the unknown location $\left(x_{c}, y_{c}\right)$ of the object. 


\subsection{Sensor Measurements}

The field generated by a radioactive substance is often described as an inhomogeneous spatial Poisson process [39]. For a localized source of radiation, the average rate of radioactive decay in space is a function of the distance to the object, and is readily described by the Inverse Square Law[40]. Thus, the intensity of the spatial Poisson process evaluated at location of the $i^{\text {th }}$ sensor is described by the following equation:

$$
\lambda\left(x_{i}, y_{i}\right)=\frac{\Lambda}{1+\left(x_{i}-x_{c}\right)^{2}+\left(y_{i}-y_{c}\right)^{2}},
$$

where $\Lambda$ is the average intensity of the field at the target's location, $\left(x_{i}, y_{i}\right)$ is the $i^{\text {th }}$ sensor location, and $\left(x_{c}, y_{c}\right)$ is the target's location. We model the measurement by the $i^{\text {th }}$ sensor as

$$
r\left(x_{i}, y_{i}\right)=n\left(x_{i}, y_{i}\right)+w\left(x_{i}, y_{i}\right)
$$

where $n\left(x_{i}, y_{i}\right)$ is the measurement of the radioactive field at the location of the $i^{\text {th }}$ sensor. It is a Poisson random variable with the probability density function $(p d f)$ :

$$
f_{N_{i}}(\alpha)=\sum_{k=0}^{\infty} \frac{\lambda\left(x_{i}, y_{i}\right)^{k}}{k !} e^{-\lambda\left(x_{i}, y_{i}\right)} \delta(\alpha-k) .
$$

and $w\left(x_{i}, y_{i}\right)$ is the additive noise (observation noise). We assume that the observation noise at $\left(x_{i}, y_{i}\right)$ is normally distributed with zero mean and variance $\sigma_{0}^{2}, N\left(0, \sigma_{0}^{2}\right)$. Its pdf is given by:

$$
f_{W_{i}}(w)=\frac{1}{\sqrt{2 \pi \sigma_{0}^{2}}} e^{-\frac{w^{2}}{2 \sigma_{0}^{2}}} .
$$

The noise measurements are independent and also independent of sensors' measurements.

\subsection{Local Processing for Digital Case}

To save the bandwidth and power during transmission[41], we quantize sensor measurements to $M$ levels by using uniform quantization[42]. Denote by $Q_{q}\left(r_{i}\right)=q_{i}$ quantized version of the $i^{\text {th }}$ measurement. Then the Fusion Center receives $K$ noisy measurements $z_{i}=q_{i}+v_{i} i=1 \ldots K$, where $v_{i}$ is the noise in the $i^{t h}$ communication channel. In this work 
we assume that $v_{i}$ are independent Gaussian random variables with zero mean and variance $\sigma_{c}^{2}$

\subsection{Problem Statement}

Given the number of sensors $K$ in the area of observation $\Omega$, the locations $\left(x_{i}, y_{i}\right)$ of the sensors, and a parametric stochastic field generated by object of interest, our goal is to estimate the location parameter $\left(x_{c}, y_{c}\right)$ and continuously track the object in time as the FC receives new sets of sensor measurements every $T$ seconds.

\subsection{Proposed Solution}

In this work, we apply a classical Maximum Likelihood Estimation approach [43], [44] to solve the problem of distributed target location estimation. This solution suggests evaluating the joint $p d f$ of the measurements at the $\mathrm{FC}$ and finding the target location, which maximizes this density. Our tracking algorithm uses the estimate of the location parameters at time $t$ as a guessing point to estimate the location of the object at time $t+T$.

\subsection{Maximum Likelihood Solution}

\subsubsection{Models for the Analog Case}

Since the random variables in (3.3) and (3.4) are independent, $p d f$ of $R_{i}$ can be obtained as a convolution $f_{R_{i}}(r)=f_{N_{i}}(r) * f_{W_{i}}(r)$ where $*$ stands for convolution, Therefore $f_{R_{i}}(r)=$ $\int_{-\infty}^{\infty} f_{N_{i}}(\alpha) f_{W_{i}}(r-\alpha) d \alpha$.

From equation (3.3) $f_{R_{i}}(r)=\sum_{k=0}^{\infty} \frac{\lambda\left(x_{i}, y_{i}\right)^{k}}{k !} e^{-\lambda\left(x_{i}, y_{i}\right)} \int_{-\infty}^{\infty} \delta(\alpha-k) f_{W_{i}}(r-\alpha) d \alpha$.

and the resulting $p d f$ is given by:

$$
f_{R_{i}}(r)=\sum_{k=0}^{\infty} \frac{\lambda\left(x_{i}, y_{i}\right)^{k}}{k !} e^{-\lambda\left(x_{i}, y_{i}\right)} f_{W_{i}}(r-k) .
$$

After substituting (3.4) into (3.5) we obtain:

$$
f_{R_{i}}(r)=\sum_{k=0}^{\infty} \frac{\lambda\left(x_{i}, y_{i}\right)^{k}}{k !} e^{-\lambda\left(x_{i}, y_{i}\right)} \frac{1}{\sqrt{2 \pi \sigma_{0}^{2}}} e^{-\frac{(r-k)^{2}}{2 \sigma_{0}^{2}}} .
$$


The $p d f$ of the measurements at the $\mathrm{FC}$ is the result of the convolution between the $p d f$ in (3.6) and the $p d f$ of the channel noise $v_{i}$, which is described as a Gaussian distributed random variable $N\left(0, \sigma_{c}^{2}\right)$. Therefore, the $p d f$ of $Z_{i}$ is given as:

$$
\begin{aligned}
f_{Z_{i}}(z) & =f_{R_{i}}(z) * f_{V_{i}}(z), \\
& =\left(\sum_{k=0}^{\infty} \frac{\lambda\left(x_{i}, y_{i}\right)^{k}}{k !} e^{-\lambda\left(x_{i}, y_{i}\right)} \frac{1}{\sqrt{2 \pi \sigma_{0}^{2}}} e^{-\frac{(z-k)^{2}}{2 \sigma_{0}^{2}}}\right) *\left(\frac{1}{\sqrt{2 \pi \sigma_{c}^{2}}} e^{-\frac{z^{2}}{2 \sigma_{c}^{2}}}\right) \\
& =\sum_{k=0}^{\infty} \frac{\lambda\left(x_{i}, y_{i}\right)^{k}}{k !} e^{-\lambda\left(x_{i}, y_{i}\right)} \int_{-\infty}^{\infty} \frac{1}{\sqrt{2 \pi \sigma_{0}^{2}}} e^{-\frac{(z-\alpha-k)^{2}}{2 \sigma_{0}^{2}}} \frac{1}{\sqrt{2 \pi \sigma_{c}^{2}}} e^{-\frac{\alpha^{2}}{2 \sigma_{c}^{2}}} d \alpha \\
& =\sum_{k=0}^{\infty} \frac{\lambda\left(x_{i}, y_{i}\right)^{k}}{k !} e^{-\lambda\left(x_{i}, y_{i}\right)} \frac{1}{\sqrt{2 \pi \sigma_{t}^{2}}} e^{-\frac{(z-k)^{2}}{2 \sigma_{t}^{2}}} \int_{-\infty}^{\infty} \frac{1}{\sqrt{2 \pi\left(\frac{\sigma_{0} \sigma_{c}}{\sigma_{t}}\right)^{2}}} e^{\left[\frac{\alpha-\frac{\sigma_{0}(z-k)^{2}}{\sigma_{t}^{2}}}{2\left(\frac{\sigma_{c} \sigma_{t}}{\sigma_{t}}\right)^{2}}\right]} d \alpha .
\end{aligned}
$$

The expression under the integral is another Gaussian $p d f$. Therefore, the integral is equal to 1 . Then the $p d f$ of $Z_{i}$ is given as:

$$
f_{Z_{i}}(z)=\sum_{k=0}^{\infty} \frac{\lambda\left(x_{i}, y_{i}\right)^{k}}{k !} e^{-\lambda\left(x_{i}, y_{i}\right)} \frac{1}{\sqrt{2 \pi \sigma_{t}^{2}}} e^{-\frac{(z-k)^{2}}{2 \sigma_{t}^{2}}}
$$

where $\sigma_{t}^{2}=\sigma_{0}^{2}+\sigma_{c}^{2}[45]$.

\subsubsection{Maximum Likelihood Estimation (analog case)}

To estimate unknown parameters, we take the maximum likelihood $(M L)$ estimation approach. Since observed data are independent, the joint pdf of vector observation $\mathbf{Z}$ is written as a product of marginal $p d f s$ :

$$
f_{Z_{1} \ldots Z_{K}}\left(z_{1} \ldots z_{K} \mid\left(x_{c}, y_{c}\right)\right)=\prod_{i=1}^{K} f_{Z_{i}}\left(z \mid\left(x_{c}, y_{c}\right)\right) .
$$

We can also apply a monotonic function such as $\log$ to the joint $p d f$ of $\mathbf{Z}$ and find its maximizer

$$
\begin{aligned}
l\left(x_{c}, y_{c}\right) & =\log \left[\prod_{i=1}^{K} f_{Z_{i}}\left(z_{i} \mid\left(x_{c}, y_{c}\right)\right]\right. \\
& =\sum_{i=1}^{K} \log \left[f_{Z_{i}}\left(z_{i} \mid\left(x_{c}, y_{c}\right)\right]\right.
\end{aligned}
$$


Substituting $f_{Z_{i}}(z)$ from (3.7) into the above expression, we obtain:

$$
l\left(x_{c}, y_{c}\right)=\sum_{i=1}^{K} \log \left[\sum_{k=0}^{\infty} \frac{\lambda\left(x_{i}, y_{i}\right)^{k}}{k !} e^{-\lambda\left(x_{i}, y_{i}\right)} \frac{1}{\sqrt{2 \pi \sigma_{t}^{2}}} e^{-\frac{\left(z_{i}-k\right)^{2}}{2 \sigma_{t}^{2}}}\right]
$$

The necessary condition for an interior maximizer of (3.9) is

$$
\left.\frac{\partial l\left(x_{c}, y_{c}\right)}{\partial x_{c}}\right|_{\hat{x}, \hat{y}}=0,\left.\frac{\partial l\left(x_{c}, y_{c}\right)}{\partial y_{c}}\right|_{\hat{x}, \hat{y}}=0 .
$$

Since $\lambda\left(x_{c}, y_{c}\right)$ is a function of $\left(x_{i}, y_{i}\right)$, the derivative of log-likelihood function in (3.9) with respect to $x_{c}$ is equal to the derivative of $l\left(x_{c}, y_{c}\right)$ with respect to $\lambda\left(x_{i}, y_{i}\right)$ multiplied by the derivative of $\lambda\left(x_{i}, y_{i}\right)$ with respect to $x_{c}$ (chain rule [46]). The same method is considered for the derivative of the log-likelihood function in (3.9) with respect to $y_{c}$. Thus,

$$
\frac{\partial l\left(x_{c}, y_{c}\right)}{\partial x_{c}}=\frac{\partial l_{Z_{1} \ldots . Z_{k}}}{\partial \lambda\left(x_{i}, y_{i}\right)} \frac{\partial \lambda\left(x_{i}, y_{i}\right)}{\partial x_{c}}
$$

and

$$
\frac{\partial l\left(x_{c}, y_{c}\right)}{\partial y_{c}}=\frac{\partial l_{Z_{1} \ldots . Z_{k}}}{\partial \lambda\left(x_{i}, y_{i}\right)} \frac{\partial \lambda\left(x_{i}, y_{i}\right)}{\partial y_{c}}
$$

where

$$
\frac{\partial \lambda\left(x_{i}, y_{i}\right)}{\partial x_{c}}=2\left(x_{i}-x_{c}\right) \lambda^{2}\left(x_{i}, y_{i}\right) / \Lambda
$$

and

$$
\frac{\partial l\left(x_{c}, y_{c}\right)}{\partial \lambda\left(x_{i}, y_{i}\right)}=\sum_{i=1}^{K} \frac{\sum_{k=0}^{\infty}\left(\frac{k \lambda\left(x_{i}, y_{i}\right)^{k-1}}{k !} e^{-\frac{\left(z_{i}-k\right)^{2}}{2 \sigma_{t}^{2}}}-\frac{\lambda\left(x_{i}, y_{i}\right)^{k}}{k !} e^{-\frac{\left(z_{i}-k\right)^{2}}{2 \sigma_{t}^{2}}}\right)}{\sum_{k=0}^{\infty} \frac{\lambda\left(x_{i}, y_{i}\right)^{k}}{k !} e^{-\frac{\left(z_{i}-k\right)^{2}}{2 \sigma_{t}^{2}}}}
$$

Substituting the above expression and (3.12) in (3.11), we obtain:

$$
\frac{\partial l\left(x_{c}, y_{c}\right)}{\partial x_{c}}=2 \sum_{i=1}^{K} \frac{\sum_{k=0}^{\infty}\left(\frac{k \lambda\left(x_{i}, y_{i}\right)^{k+1}}{k !}-\frac{\lambda\left(x_{i}, y_{i}\right)^{k+2}}{k !}\right) e^{-\frac{\left(z_{i}-k\right)^{2}}{2 \sigma_{t}^{2}}}\left(x_{i}-x_{c}\right)}{\Lambda \sum_{k=0}^{\infty} \frac{\lambda\left(x_{i}, y_{i}\right)^{k}}{k !} e^{-\frac{\left(z_{i}-k\right)^{2}}{2 \sigma_{t}^{2}}}}
$$

The same procedure is applied to determine the derivative with respect to $y_{c}$ :

$$
\frac{\partial l\left(x_{c}, y_{c}\right)}{\partial y_{c}}=2 \sum_{i=1}^{K} \frac{\sum_{k=0}^{\infty}\left(\frac{k \lambda\left(x_{i}, y_{i}\right)^{k+1}}{k !}-\frac{\lambda\left(x_{i}, y_{i}\right)^{k+2}}{k !}\right) e^{-\frac{\left(z_{i}-k\right)^{2}}{2 \sigma_{t}^{2}}}\left(y_{i}-y_{c}\right)}{\Lambda \sum_{k=0}^{\infty} \frac{\lambda\left(x_{i}, y_{i}\right)^{k}}{k !} e^{-\frac{\left(z_{i}-k\right)^{2}}{2 \sigma_{t}^{2}}}}
$$




\subsubsection{Models for the Digital Case}

The digital case assumes that a sensor measurement $r_{i}$ is sent to a $M$-level uniform quantizer[42] where the sensory data are quantized to $M$ quantization levels, with reproduction points denoted as $\left(\gamma_{1}, \gamma_{2} \ldots \gamma_{M}\right)$. We assume that quantizer is deterministic. Denote the output of the quantizer as $q_{i}=Q_{q}\left(r_{i}\right)$.

The probability of the $j^{\text {th }}$ reproduction point is:

$$
P_{i j}=\int_{\tau_{j}}^{\tau_{j+1}} f_{R_{i}}(r) d r
$$

where $\tau_{j}, j=1 \ldots M-1$ are the boundaries of quantization regions. After substituting (3.6) in place of $f_{R_{i}}(r)$ :

$$
P_{i j}=\sum_{k=0}^{\infty} \frac{\lambda\left(x_{i}, y_{i}\right)^{k}}{k !} e^{-\lambda\left(x_{i}, y_{i}\right)} \int_{\tau_{j}}^{\tau_{j+1}} \frac{1}{\sqrt{2 \pi \sigma_{0}^{2}}} e^{-\frac{(r-k)^{2}}{2 \sigma_{0}^{2}}} d r
$$

In terms of $Q$-functions[47], (3.15) becomes:

$$
P_{i j}=\sum_{k=0}^{\infty} \frac{\lambda\left(x_{i}, y_{i}\right)^{k}}{k !} e^{-\lambda\left(x_{i}, y_{i}\right)}\left[Q\left(\frac{\left(k-\tau_{j+1}\right)}{\sigma_{0}}\right)-Q\left(\frac{\left(k-\tau_{j}\right)}{\sigma_{0}}\right)\right] .
$$

where $Q(x)$ is defined as

$$
Q(x)=\frac{1}{\sqrt{2 \pi}} \int_{x}^{\infty} e^{-\frac{u^{2}}{2}} d u
$$

The quantized measurement $q_{i}$ is a discrete random variable, with generalized $p d f$ given as:

$$
f_{q_{i}}(\alpha)=\sum_{j=1}^{M} P_{i j} \delta\left(\alpha-\gamma_{j}\right)
$$

Sensors communicate their quantized observations to the Fusion Center for a joint processing. We assume that there is no noise in channels, only white Gaussian noise is added at the receiver end. This assumption results in the following model for the observed data at the FC $z_{i}=q_{i}+v_{i}, i=1 \ldots K$. Since the observations and additive noise are independent random variables, the $p d f$ of $Z_{i}$ is:

$$
f_{Z_{i}}(z)=f_{Q_{i}}(z) * f_{V_{i}}(z)
$$


where " *" stands for convolution. Substituting (3.17) into (3.18) and performing the convolution we obtain:

$$
\begin{aligned}
f_{Z_{i}}(z) & =\sum_{j=1}^{M} P_{i j}\left(\delta\left(z-\gamma_{j}\right) * f_{V_{i}}(z)\right) . \\
& =\sum_{j=1}^{M} P_{i j}\left(\int_{-\infty}^{\infty} \delta\left(\alpha-\gamma_{j}\right) f_{V_{i}}(z-\alpha)\right) d \alpha . \\
& =\sum_{j=1}^{M} P_{i j} f_{V_{i}}\left(z-\gamma_{j}\right) .
\end{aligned}
$$

Thus,

$$
f_{Z_{i}}(z)=\sum_{j=1}^{M} P_{i j} \frac{1}{\sqrt{2 \pi \sigma_{c}^{2}}} e^{\frac{\left(z-\gamma_{j}\right)^{2}}{\sigma_{c}^{2}}} .
$$

Substituting (3.16) in the above equation results in:

$$
f_{Z_{i}}(z)=\sum_{j=1}^{M} \sum_{k=0}^{\infty} \frac{\lambda\left(x_{i}, y_{i}\right)^{k}}{k !} e^{-\lambda\left(x_{i}, y_{i}\right)}\left[Q\left(\frac{\left(k-\tau_{j+1}\right)}{\sigma_{0}}\right)-Q\left(\frac{\left(k-\tau_{j}\right)}{\sigma_{0}}\right)\right] \frac{1}{\sqrt{2 \pi \sigma_{c}^{2}}} e^{-\frac{\left(z-\gamma_{j}\right)^{2}}{2 \sigma_{c}^{2}}} .
$$

\subsubsection{Maximum Likelihood Estimation (digital case)}

Our approach to solve for unknown parameters of the field is similar to that of analog channel case. The joint log-likelihood function of the data observed by the FC is given by:

$$
\begin{aligned}
l\left(x_{c}, y_{c}\right) & =\log \prod_{i=1}^{K} f_{Z_{i}}\left(z_{i}\right) \\
& =\sum_{i=1}^{K} \log \left(f_{Z_{i}}\left(z_{i}\right)\right) .
\end{aligned}
$$

Substituting (3.20) into the log-likelihood function, we obtain:

$$
l\left(x_{c}, y_{c}\right)=\sum_{i=1}^{K} \log \left[\sum_{j=1}^{M} \sum_{k=0}^{\infty} \frac{\lambda\left(x_{i}, y_{i}\right)^{k}}{k !} e^{-\lambda\left(x_{i}, y_{i}\right)} \Delta Q_{k, j} \frac{1}{\sqrt{2 \pi \sigma_{c}^{2}}} e^{-\frac{\left(z_{i}-\gamma_{j}\right)^{2}}{2 \sigma_{c}^{2}}}\right] .
$$

Where $\Delta Q_{k, j}$ is given as:

$$
\left[Q\left(\frac{\left(k-\tau_{j+1}\right)}{\sigma_{0}}\right)-Q\left(\frac{\left(k-\tau_{j}\right)}{\sigma_{0}}\right)\right]
$$


The $M L$ estimation approach is seeking for the pair of parameters $\left(x_{c}, y_{c}\right)$ that maximizes (3.21). If $l_{Z_{1} \ldots Z_{K}}$ is continuous function in the vicinity of $\left(x_{c}, y_{c}\right)$ and the optimal $\left(x_{c}, y_{c}\right)$ is the inner point in $\Omega$, the necessary conditions for a maximizer are:

$$
\left.\frac{\partial l\left(x_{c}, y_{c}\right)}{\partial x_{c}}\right|_{\hat{x}, \hat{y}}=\left.\frac{\partial l\left(x_{c}, y_{c}\right)}{\partial \lambda\left(x_{i}, y_{i}\right)} \frac{\partial \lambda\left(x_{i}, y_{i}\right)}{\partial x_{c}}\right|_{\hat{x}, \hat{y}}=0
$$

and

$$
\left.\frac{\partial l\left(x_{c}, y_{c}\right)}{\partial y_{c}}\right|_{\hat{x}, \hat{y}}=\left.\frac{\partial l\left(x_{c}, y_{c}\right)}{\partial \lambda\left(x_{i}, y_{i}\right)} \frac{\partial \lambda\left(x_{i}, y_{i}\right)}{\partial y_{c}}\right|_{\hat{x}, \hat{y}}=0
$$

Here,

$$
\frac{\partial l\left(x_{c}, y_{c}\right)}{\partial \lambda\left(x_{c}, y_{c}\right)}=\sum_{i=1}^{K} \frac{\sum_{j=1}^{M} \sum_{k=0}^{\infty}\left(k \frac{\lambda\left(x_{i}, y_{i}\right)^{k-1}}{k !}-\frac{\lambda\left(x_{i}, y_{i}\right)^{k}}{k !}\right) \Delta Q_{k, j} e^{-\frac{\left(z-\gamma_{j}\right)^{2}}{2 \sigma_{c}^{2}}}}{\sum_{j=1}^{M} \sum_{k=0}^{\infty} \frac{\lambda\left(x_{i}, y_{i}\right)^{k}}{k !} \Delta Q_{k, j} e^{-\frac{\left(z-\gamma_{j}\right)^{2}}{2 \sigma_{0}^{2}}}}
$$

and after substituting equation (3.12) and the above formula into equation (3.22):

$$
\frac{\partial l\left(x_{c}, y_{c}\right)}{\partial x_{c}}=2 \sum_{i=1}^{K} \frac{\sum_{j=1}^{M} \sum_{k=0}^{\infty}\left(k \frac{\lambda\left(x_{i}, y_{i}\right)^{k+1}}{k !}-\frac{\lambda\left(x_{i}, y_{i}\right)^{k+2}}{k !}\right) \Delta Q_{k, j} e^{-\frac{\left(z-\gamma_{j}\right)^{2}}{2 \sigma_{c}^{2}}}\left(x_{i}-x_{c}\right)}{\Lambda \sum_{j=1}^{M} \sum_{k=0}^{\infty} \frac{\lambda\left(x_{i}, y_{i}\right)^{k}}{k !} \Delta Q_{k, j} e^{-\frac{\left(z-\gamma_{j}\right)^{2}}{2 \sigma_{0}^{2}}}} .
$$

Similarly,

$$
\frac{\partial l\left(x_{c}, y_{c}\right)}{\partial y_{c}}=2 \sum_{i=1}^{K} \frac{\sum_{j=1}^{M} \sum_{k=0}^{\infty}\left(k \frac{\lambda\left(x_{i}, y_{i}\right)^{k+1}}{k !}-\frac{\lambda\left(x_{i}, y_{i}\right)^{k+2}}{k !}\right) \Delta Q_{k, j} e^{-\frac{\left(z-\gamma_{j}\right)^{2}}{2 \sigma_{c}^{2}}}\left(y_{i}-y_{c}\right)}{\Lambda \sum_{j=1}^{M} \sum_{k=0}^{\infty} \frac{\lambda\left(x_{i}, y_{i}\right)^{k}}{k !} \Delta Q_{k, j} e^{-\frac{\left(z-\gamma_{j}\right)^{2}}{2 \sigma_{0}^{2}}}} .
$$

The equations (3.24) and (3.25) are highly nonlinear in $\left(x_{c}, y_{c}\right)$ and cannot be solved in closed form. In the next section, we propose an iterative solution to the problem and in Chapter 4 we analyze its performance by using Monte Carlo simulation method[48].

\subsection{Iterative solution}

The proposed solution is an effective combination of two known numerical methods to solve for the roots $\left(x_{c}, y_{c}\right)$ of the above nonlinear equations $(3.13),(3.14),(3.24)$ and (3.25). The two introduced methods to solve the nonlinear equations are Bisection and Secant [49] 
[50],[51] . Suppose we have a task to find an optimizer of a function $f(x)$, a real-valued one dimensional function. Bisection method chooses two guessing points $a$ and $b$ at every iteration such that $f(a)$ and $f(b)$ have the opposite signs. This guarantees that the root lies within $[a, b]$. A third point $m$ is calculated as the half way between $a$ and $b$, that is $m=\frac{a+b}{2}$. The sign of $f(m)$ is compared to both $f(a)$ and $f(b)$. If $f(m)$ and $f(a)$ have the opposite signs, then the point $m$ replaces the point $b$, otherwise, $f(m)$ will have the opposite sign with $f(b)$, and $m$ replaces the point $a$. Iterations continue until a tolerant value is reached. A block-diagram of the algorithm is displayed in Figure (3.2). 


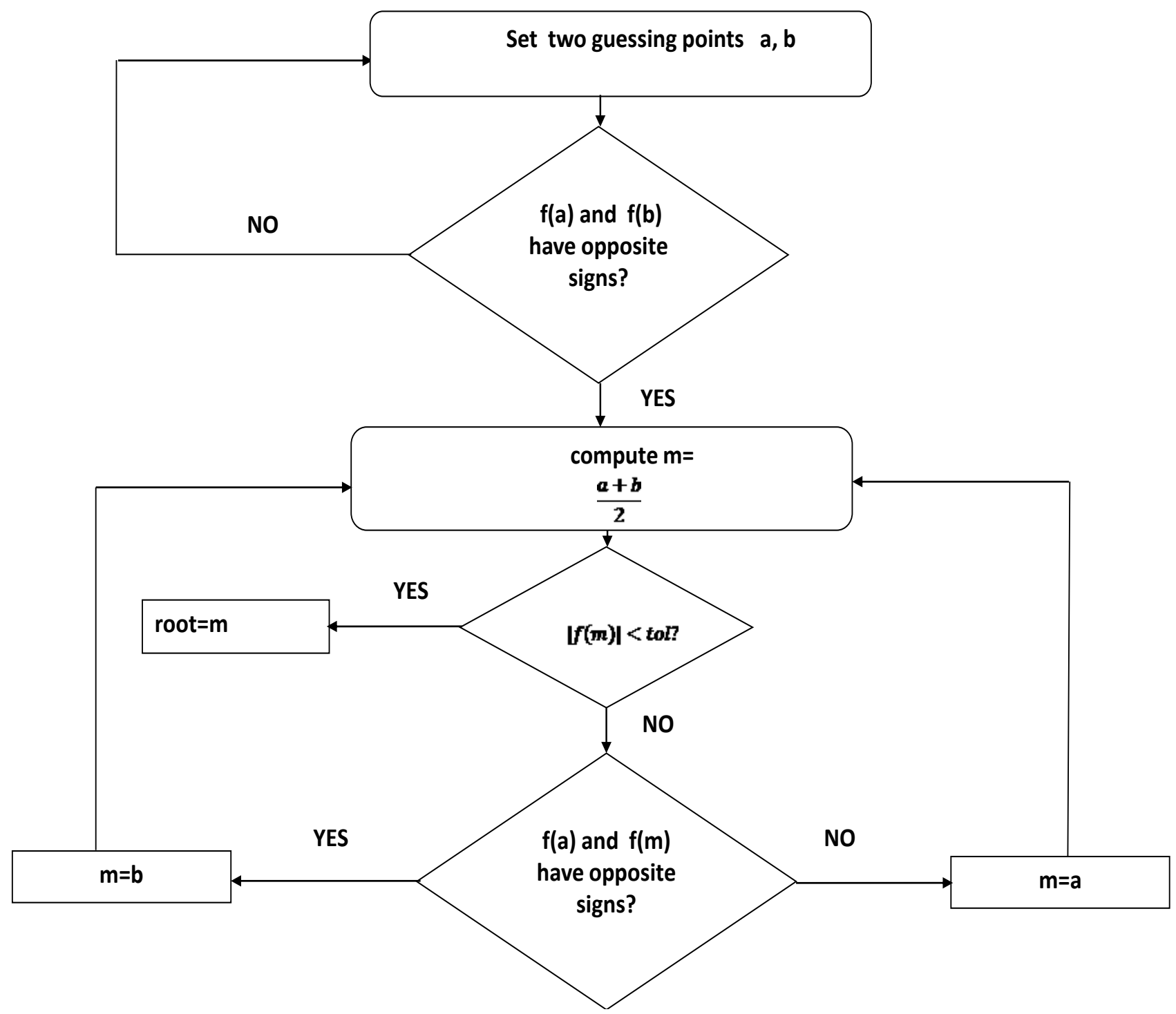

Figure 3.1: A block diagram of the Bisection method.

The second approach, Secant method, also assumes initialization. Two guessing points are used to evaluate the following function at every iteration:

$$
S=\text { guessing }_{n-1}-f\left(\text { guessing }_{n-1}\right) \frac{\text { guessing }_{n-1}-\text { guessing }_{n-2}}{f\left(\text { guessing }_{n-1}\right)-f\left(\text { guessing }_{n-2}\right)} .
$$

Iterations continue until a point of convergence is reached.

Our implementation combines the Secant and Bisection methods leading to an iterative method that has the reliability and precision of Bisection rule and the speed of Secant 


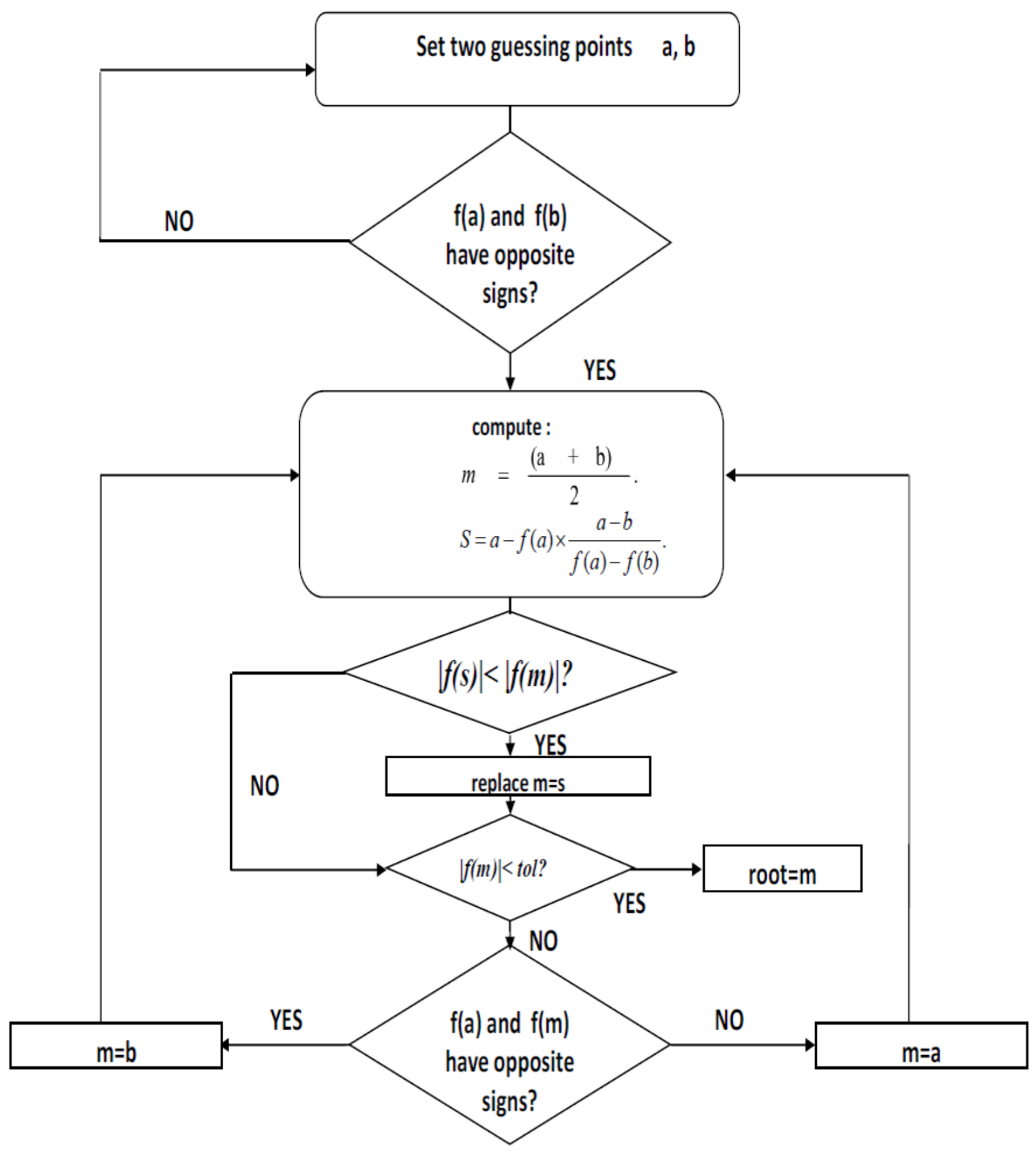

Figure 3.2: The combination of the two methods, Bisection and Secant. 
method. This combination is due to Dekker[20] [21], [22]. The same guessing points are applied in both methods to compare between the two outputs at each iteration. The goal at each iteration is to find out which is the closer point to the root, $m$ or $S$. Here $|f(m)|$ is compared with $|f(s)|$. If $|f(s)|$ is less than $|f(m)|$, the point $s$ is the better guess than $m$, and it is likely closer to the root than the point $m$. It will be then taken as one of the two guesses in the next bisection iteration. If not, then the point $m$ will take place. Figure 3.2 presents the combination between the two methods, and it is illustrated that the point $m$ is the result of each iteration and to be substituted in the function to check whether the convergence is reached. Since our functions have two roots, the algorithms are run separately and simultaneously for each root. At every iteration both resulting $m$ values of the two iterations are substituted into the function, and the result is compared with the tolerant value to estimate the roots. 


\section{Chapter 4}

\section{Numerical Results}

In this chapter we present numerical results that test the accuracy of the algorithm proposed in Chapter 3 and evaluate the effect of various parameters on the performance of the developed algorithm in both analog and digital cases.

For our simulation, we constrain the area of sensor network denoted as $\Omega$ to a square of size $2 \times 2$. A set of $K$ sensors are randomly positioned within the area, and the locations of sensors are noted. We generate data received by the FC by means of Monte Carlo simulations [48] [52]. To accumulate statistics, we generate 1000 realizations of a single experiment. Each Monte Carlo realization of the data at the FC is obtained by:

1. Taking samples of a randomly generated Poisson field located at $\left(x_{c}, y_{c}\right)$ with the spatial intensity given as:

$$
\lambda(x, y)=\frac{\Lambda}{1+\left(x-x_{c}\right)^{2}+\left(y-y_{c}\right)^{2}},
$$

at $K$ sensor locations.

2. Adding realizations of Gaussian noise with zero mean and variance $\sigma_{0}^{2}$.

3. Further applying a deterministic quantizer to the samples in item (2) (in case of digital channel) or keeping samples as they are (in the case of analog channel).

4. Generating and adding realizations of Gaussian noise with zero mean and variance $\sigma_{c}^{2}$ to the samples in item 3. The values of Signal-to-Noise ratios $(S N R s)$ are determined by variances $\sigma_{0}^{2}$ and $\sigma_{c}^{2}$ in observation and transmission channels. In our simulations, 
$K, M, S N R_{0}$ and $S N R_{c}$ are parameters. The original field in the simulations is positioned at $\left(x_{c}, y_{c}\right)$.

Once the data are generated, we apply the algorithm described in Chapter 3 to find the $M L$ estimates of the field location.

As a measure of estimation performance we choose Square Error $(S E)$, defined as:

$$
S E=\left(\hat{x}_{c}-x_{c}\right)^{2}+\left(\hat{y}_{c}-y_{c}\right)^{2}
$$

where $\hat{x}_{c}$ and $\hat{y}_{c}$ are $M L$ estimates of the location parameters $\left(x_{c}, y_{c}\right)$ of the field based on a single Monte Carlo realization of observations at the FC.

Many results in this chapter will be presented as a box plot of $S E$ displayed as a function of one of parameters. A box plot is a scatter plot of a set of points displayed as a box with the bar inside it indicating the median of the values of the points in the set. The boundaries of the box are 25-th and 75-th quantiles of values that points in the set take. The outliers points are marked with "+" sign.

Section 4.1 defines Signal-to-Noise Ratio $S N R$ in observation and transmission channels. These $S N R s$ are then used both as performance measures and as parameters of the considered WSN.

Sections 4.2 and 4.3 describe a set of experiments demonstrating performance of the iterative estimator introduced in Chapter 3.

\subsection{Signal to Noise Ratio}

In this subsection we define Signal-to-Noise Ratio in observation channel and transmission channel.

Observation channel is due to additive noise at the receiver end of each sensor. Each output antenna adds white Gaussian noise to a sample of the field.

Transmission channel is the same as communication channel. Here we assume that sensor measurements are communicated over distortion-free environment (no attenuation, no distortion or interference), but the receiver end of the channel (receiver antenna) adds white 
Gaussian noise. This noise is independent of the noise in observation channels.

To measure the robustness of the $M L$ estimator with respect to the noise power (variance) in the observation and transmission channels, we introduce $S N R_{0}, S N R$ in observation channels and $S N R_{c}, S N R$ in transmission channels of the distributed network.

\subsubsection{Observation SNR}

The observation $S N R, S N R_{0}$, is defined as the target signal power integrated over area $\Omega$, divided by the variance of the noise and by the area:

$$
S N R_{0}=\frac{E\left(N_{\Omega}^{2}\right)}{\Omega \sigma_{0}^{2}}
$$

where $E\left(N_{\Omega}^{2}\right)$ is the second moment of the random Poisson field at location $(x, y) \epsilon \Omega$. It is given as:

$$
E\left(N_{\Omega}^{2}\right)=\lambda_{\Omega}+\lambda_{\Omega}^{2}
$$

where

$$
\lambda_{\Omega}=\iint_{\Omega} \frac{\Lambda}{1+\left(x_{c}-x\right)^{2}+\left(y_{c}-y\right)^{2}} d y d x .
$$

The integration in (4.2) is performed by transforming $(x, y)$ into polar representation $(r, \theta)$ as follows:

$$
\begin{aligned}
\lambda_{\Omega} & =8 \int_{0}^{\pi / 4} \int_{1}^{1 / \cos (\theta)} \frac{\Lambda}{1+R^{2}} R d R d \theta . \\
& =\left.8 \int_{0}^{\pi / 4} \frac{\Lambda}{2} \ln \left(1+R^{2}\right)\right|_{1} ^{1 / \cos (\theta)} d \theta . \\
& =4 \Lambda\left[\int_{0}^{\pi / 4} \ln \left(1+\frac{1}{\cos ^{2}(\theta)}\right) d \theta-\frac{\pi \ln (2)}{4}\right] .
\end{aligned}
$$

The integration is performed using alpha Wolfram [53], and the $S N R_{0}$ is calculated with $\Lambda=100$ and $\sigma_{0}^{2}=0.8$, and found to be about $27 \mathrm{~dB}$. 


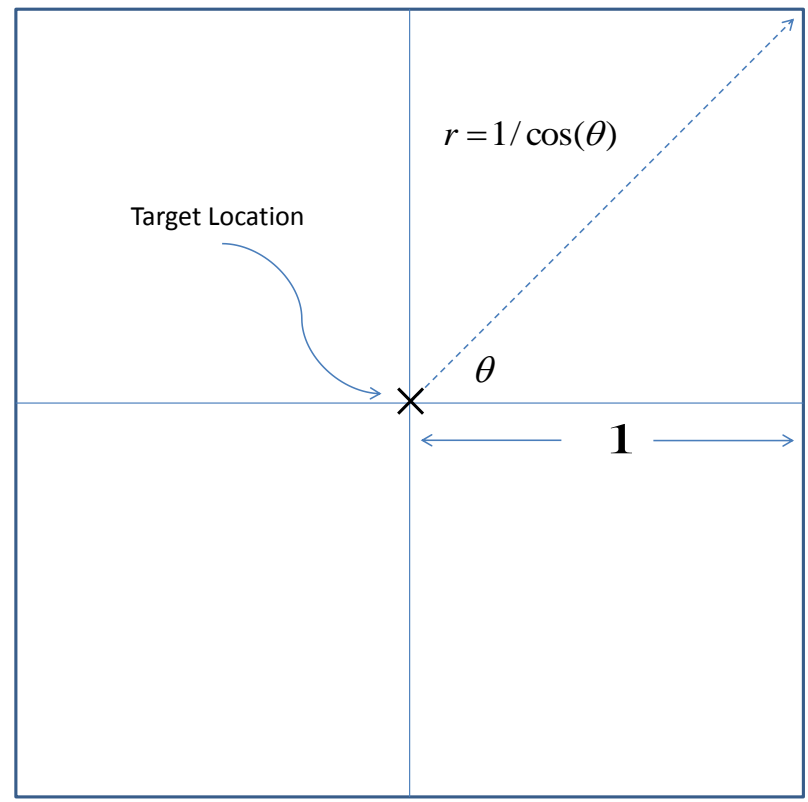

Figure 4.1: The area $\Omega$, limits of Polar Integration

\subsubsection{Transmission SNR}

We define transmission $S N R$ as:

$$
S N R_{c}=\frac{P W}{\Omega \sigma_{c}^{2}}
$$

where $P W$ is the total delivered power at the input to the communication channel. Since we perform different signal processing on the input to analog and digital channel, we have two different expressions for $P W$.

\section{Analog Case}

Since the signal power at the input to the channel depends on the number of allocated sensors in the area, $S N R_{c}$ is evaluated as the summation of powers that transferred by the sensors from the area to the channel divided by the channel noise variance $\sigma_{c}^{2}$. The signal power at the input to the channel is defined as the second moment of the random variable $r$ in the analog case. Therefore, according to equation (3.6):

$$
E\left(r^{2}\right)=\int_{-\infty}^{\infty} r^{2} f_{r}(r) d r
$$


The second moment is evaluated as follows:

$$
E\left(r^{2}\right)=\sum_{k=0}^{\infty} \frac{\lambda_{\Omega}^{k}}{k !} e^{-\lambda_{\Omega}} \int_{-\infty}^{\infty} \frac{r^{2}}{\sqrt{2 \pi \sigma_{0}^{2}}} e^{-\frac{(r-k)^{2}}{2 \sigma_{0}^{2}}} d r
$$

where $\int_{-\infty}^{\infty} \frac{r^{2}}{\sqrt{2 \pi \sigma_{0}^{2}}} e^{-\frac{(r-k)^{2}}{2 \sigma_{0}^{2}}} d r$ is the second moment of normal distribution, which is equal to $\left(k^{2}+\sigma_{0}^{2}\right)[54]$.

Therefore,

$$
E\left(r^{2}\right)=\sum_{k=0}^{\infty} \frac{\lambda_{\Omega}^{k}}{k !} e^{-\lambda_{\Omega}}\left(k^{2}+\sigma_{0}^{2}\right) .
$$

The power of the input to the channel depends on the number of sensors as well as the location of each sensor. In our case the sensors are uniformly distributed over the area $\Omega$, and the total transferred power to the channel is given by the product of the average measurement power at the expected sensor location and the number of sensors. The expected sensor location is at the center of the area, where the target field intensity is at its maximum value. Therefore, the calculated $S N R_{c}$ here is the maximum $S N R$ that could be achieved in this case. Thus,

$$
S N R_{c}=\frac{K \sum_{k=0}^{\infty} \frac{\lambda_{\Omega}^{k}}{k !} e^{-\lambda_{\Omega}}\left(k^{2}+\sigma_{0}^{2}\right)}{\Omega \sigma_{c}^{2}} .
$$

$S N R_{c}$ for the analog channel is determined under $\Lambda=100, K=100$, and $\sigma_{c}^{2}=0.8$, and found to be about $46 d B$.

\section{Digital Case}

For the digital case, we evaluate the power of quantized sensor measurements as follows:

$$
E\left(q_{i}^{2}\right)=\int_{-\infty}^{\infty} \alpha^{2} f_{Q}(\alpha) d \alpha
$$

where $q_{i}$ is the quantized measurement at the location of object, which is considered here as the center of the area.

$$
f_{q_{i}}(\alpha)=\sum_{J=1}^{M} P_{i j} \delta\left(\alpha-\gamma_{J}\right) .
$$

Thus,

$$
E\left(q_{i}^{2}\right)=\sum_{J=1}^{M} P_{i j} \int_{-\infty}^{\infty} \alpha^{2} \delta\left(\alpha-\gamma_{J}\right) d \alpha=\sum_{J=1}^{M} P_{i j} \gamma_{j}^{2} .
$$


After substituting $P_{i j}$ from equation (3.16):

$$
S N R_{c}=\frac{K \sum_{j=1}^{M} \sum_{k=0}^{\infty}\left[Q\left(\frac{\left(k-\tau_{j+1}\right)}{\sigma_{0}^{2}}\right)-Q\left(\frac{\left(k-\tau_{j}\right)}{\sigma_{0}}\right)\right] \gamma_{J}^{2} \frac{\lambda_{\Omega}^{k}}{k !} e^{-\lambda_{\Omega}}}{\Omega \sigma_{c}^{2}} .
$$

In our case, $S N R_{c}=26 d B$ as calculated by means of MATLAB. 


\subsection{Analog Channel}

This section presents few simulation results that we conducted under the assumption of analog transmission channel.

\subsubsection{Effect of sensors density on the estimation performance}

Our first experiment assumes that the coverage of the network is fixed, that is, the area $\Omega$ remains the same, but the number of sensors in the network grows. Our simulation results for this case are displayed in Figure 4.2. It presents the dependence of $S E$ on the number of sensors, $K$. For each fixed value $K, 1000$ Monte Carlo realizations of the network is implemented, and for each realization, the algorithm finds ML estimate of the location parameter. The outcome is displayed as a box plot of $S E$ versus values of $K$. As expected, the median of $S E$ as well as the spread of $S E$ around its median decreases as $K$ grows. When $K=10$, the median value of $S E$ is approximately 0.008 . When $K$ is increased to 50 , the median value of $S E$ drops below 0.003 .

\subsubsection{Initialization of iterative algorithm}

The choice of initial value in iterative ML solution is expected to affect the convergence of the ML algorithm to its stationary value. In this subsection, we illustrate the dependence of the $S E$ values on the deviation of the initial value from the true location of the object. We partition a circular area centered at the true location of the object into a set of no overlapping rings with the difference between radii of adjacent rings equal to 1 . All rings are numbered in the increasing order, that is, ring 1 has the smallest radius, and ring 5 has the largest radius. To explore convergence of the $\mathrm{ML}$ algorithm, for a given radius of the ring we generate 1000 Monte Carlo realizations of the data acquired in the network and for each realization of the network, we initialize the ML algorithm by values of $x$ and $y$ randomly selected within the ring. Figure 4.3 shows the results of our simulations. Rings are numbered 1 through 5, and the cumulative behavior of $S E$ is displayed as a function of the label of the ring. We can observe that both the median value of $S E$ and its variance grows with increased value of the label. However, this is a very slow growth. Note that the median 
value from Ring 1 to Ring 5 grows from about 0.001 to about 0.002 , only two times. The spread of $S E$ for these two cases differs by factor of 10 .

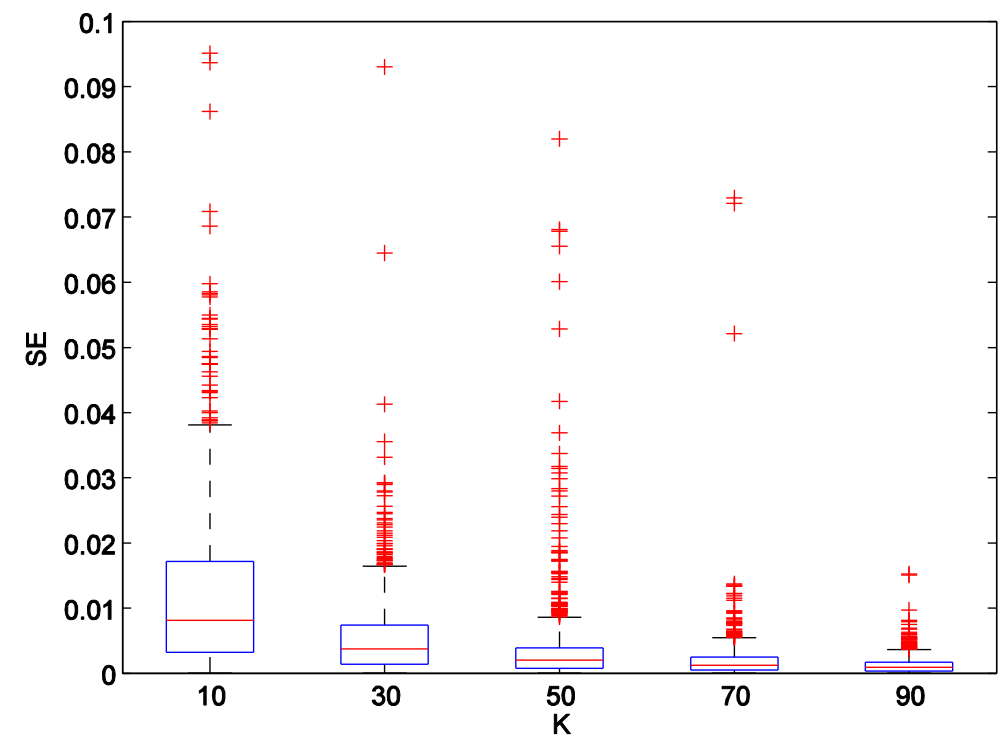

Figure 4.2: A box plot of SE as a function of the number of sensors $K$ placed within the area $\Omega$ for the analog case. $S N R_{0}=27 d B$, and $\sigma_{c}^{2}=0.8$.

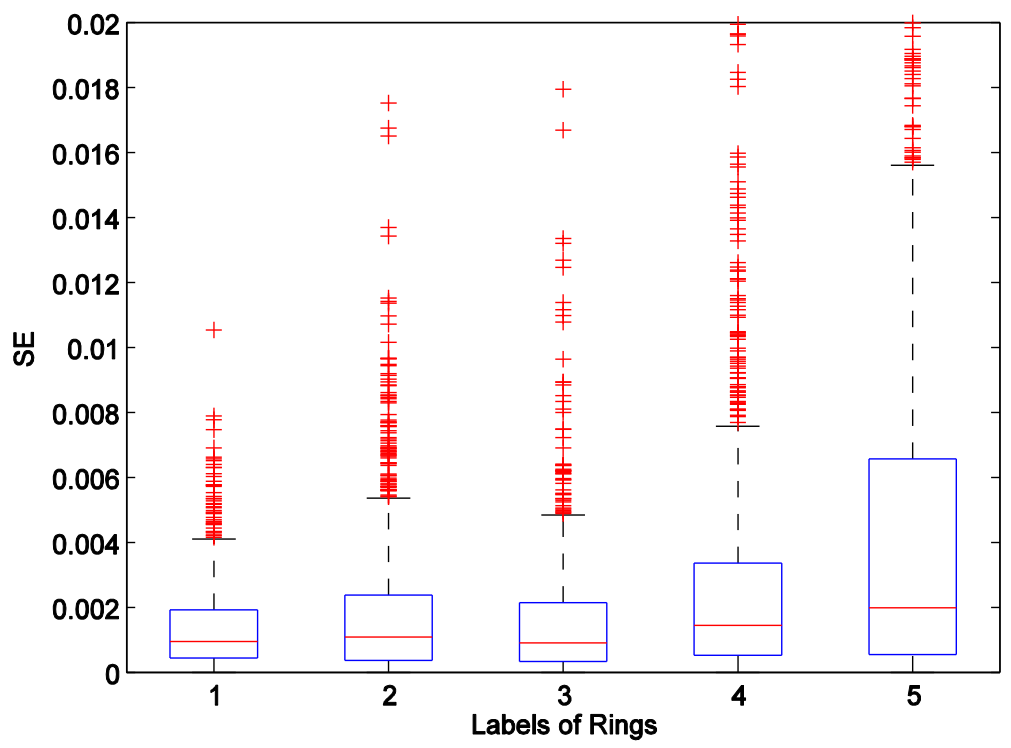

Figure 4.3: A box plot of SE as a function of a region, where initial values of estimates are drawn. $S N R_{0}=27 d B$, and $\sigma_{c}^{2}=0.8$. 


\subsubsection{Effect of the strength of the field on the estimation error}

Figure 4.4 shows the box plot of $S E$ as a function of the strength of the field, previously defined as $\Lambda$. As it is anticipated, the estimation performance improves (median $S E$ becomes smaller and has smaller variance) as the strength of the field increases. In this simulations, the noise variance in observation channel is set to $\sigma_{0}^{2}=0.8$. As we will relate it later, this plot also shows the dependence of the estimation error on the value of $S N R$ in observation channel. Later in simulating various tracking cases, we will fix $\Lambda$ at 100 for the most of the experiments.

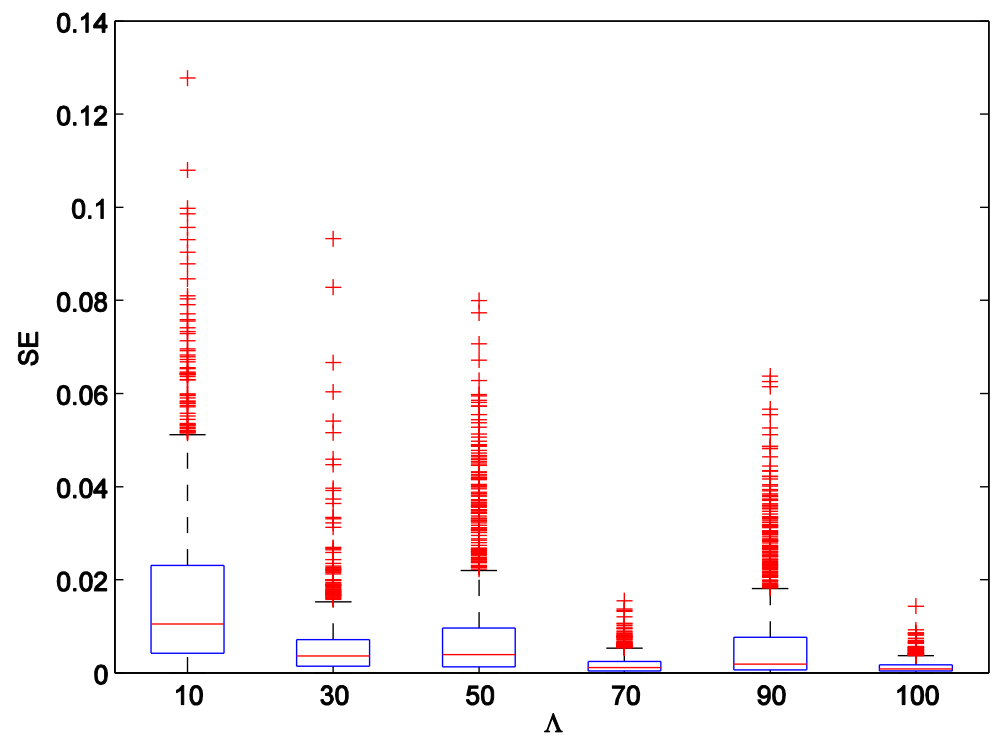

Figure 4.4: $\mathrm{SE}$ versus $\Lambda, \sigma_{0}^{2}$ is set to 0.8 , analog case. 


\subsection{Digital Channel}

This section deals with analysis of digital transmission channel.

\subsubsection{Effect of sensor density on the estimation performance: dig- ital case}

The results for this case are presented in Figure 4.5, and the dependence of $S E$ on the sensor density is demonstrated. For each given number of sensors K, 500 Monte Carlo runs are carried out. The results are displayed as a box plot of $S E$ versus values of $K$. The median of $S E$ as well as the spread of $S E$ around the median decreases as $K$ increases. When $K=10$, the median value of $S E$ is approximately 0.018 . When $K$ is increased to 50 , the median value of $S E$ drops below 0.002 and reaches approximately zero at $K \geq 70$.

\subsubsection{Effect of the number of quantization levels $M$ on the esti- mation performance}

The dependence of $S E$ on the number of quantization levels $M$ is illustrated in Figure 4.6. The experiment is run for three values of $M=8,16$, and 32 , and for each $M$ value 500 Monte Carlo runs are performed. The box plot shows an expected decrease in the $S E$ variance and median as $M$ increases.

Signal to Noise Ratio at the observation channel $S N R_{0}$ is set to $27 d B$. The sensors number $K$ is fixed to 100 sensors. $S E$ median at $M=8$ is about 0.0025 , while at $M=32$ it drops to less than 0.001 . Therefore, it could be concluded that a better performance is achieved with more quantization levels. However, computational cost grows with $M$. For large $M$, the algorithm requires more iterations, which costs more time and requires faster FC processor. 


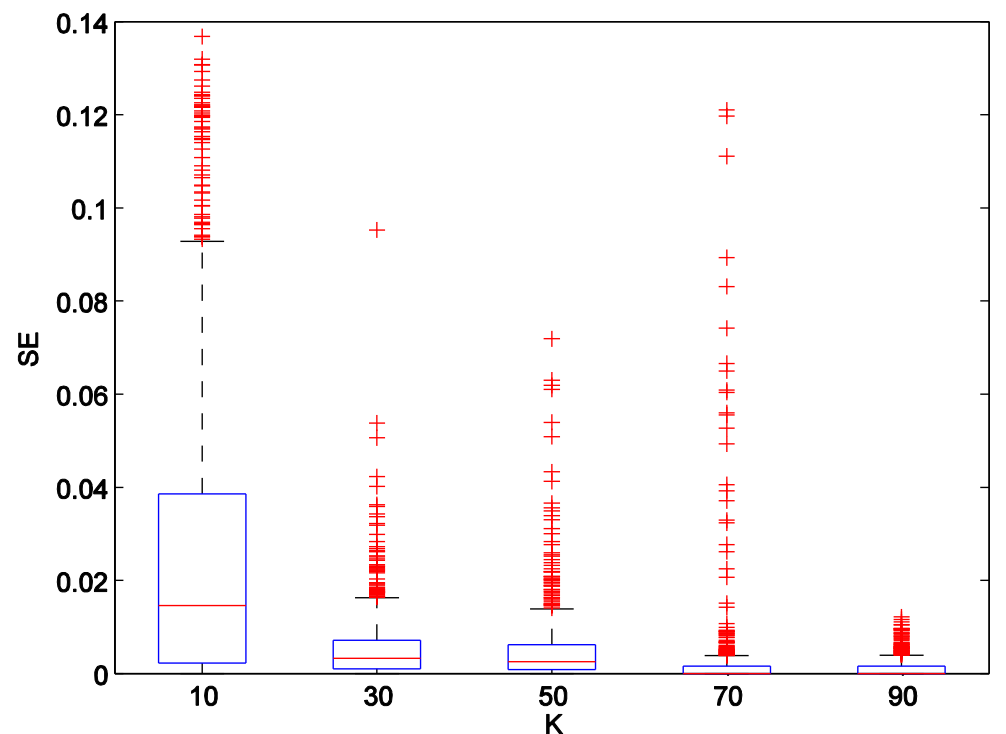

Figure 4.5: A box plot of SE as a function of the number of sensors $K$ placed within the area $\Omega$ for the digital case. $S N R_{0}=27 d B, \sigma_{c}^{2}=0.8$, and $M=16$ levels.

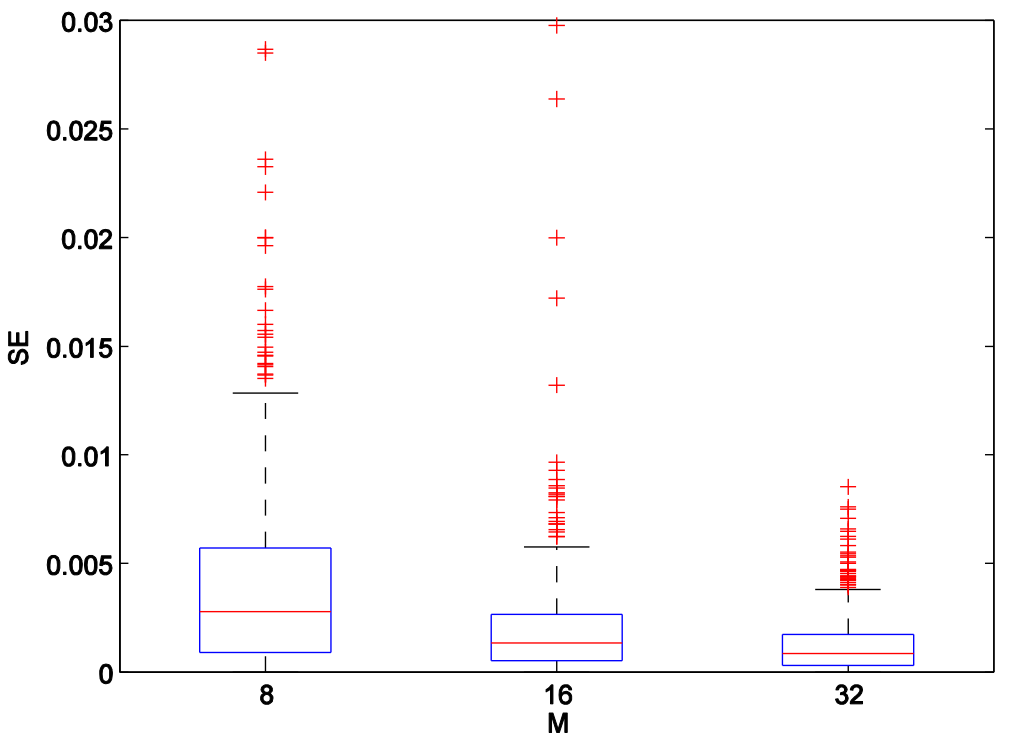

Figure 4.6: A box plot of SE as a function of the number of quantization levels $M$. $S N R_{0}=27$ $d B, \sigma_{c}^{2}=0.8$. 


\subsubsection{Initialization of iterative algorithm: digital case}

Similarly to the experiment for the analog case, we evaluate the sensitivity of the algorithm to the choice of the initial value. Instead of 5 regions we consider only 3 regions in this case. 500 Monte Carlo realizations of data are generated for each ring. Two guessing points are selected randomly from each ring at each realization. It is observed that $S E$ grows slowly as the deviation of the initial guess (from the true location) increases. Ring 1 has radius of 1 , which is the smallest radius, and the result of the simulation shows the median $S E$ of value 0.001 , while it grows to 0.009 at the ring number 3 as presented in Figure 4.7. The algorithm, as observed, is very robust with respect to the choice of initial value. This could be attributed to the part of the algorithm, where the middle between the guessing points is calculated (bisection rule). This cuts long distance by half. The middle point is then set as one of the guessing points in the next iteration. The larger deviation actually causes more iterations, and therefore costs more time. In this experiment $\sigma_{0}^{2}$ is set to 0.8 , $M=16, K=100$, and $\Lambda=100$.

\subsubsection{Effect of the strength of the field on the estimation error: digital case}

Figure 4.8 illustrates the box plot of $S E$ versus the strength of the field, $\Lambda$. As anticipated, $S E$ decreases (median $S E$ becomes smaller and has smaller spread) as the strength of the field increases, and drops from 0.008 at $\Lambda=10$ to almost 0.0005 at $\Lambda=90$. The noise variance in observation channel is set in this simulation to $\sigma_{0}^{2}=0.8$. This plot also reveals the dependence of the algorithm performance on the value of $S N R_{0}$ in observation channel, because it directly depends on the average value of the field strength, as it will be explained in Section 4.4. 


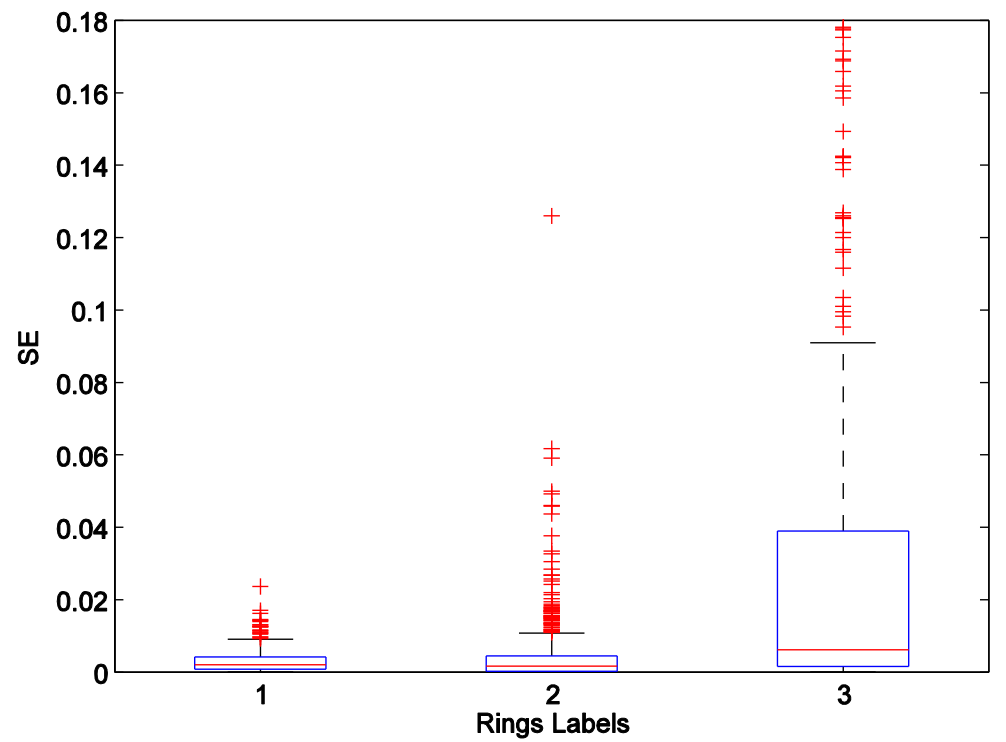

Figure 4.7: SE versus Ring Labels, $\Lambda=100, \sigma_{0}^{2}=0.8$, and $M=16$ : digital case.

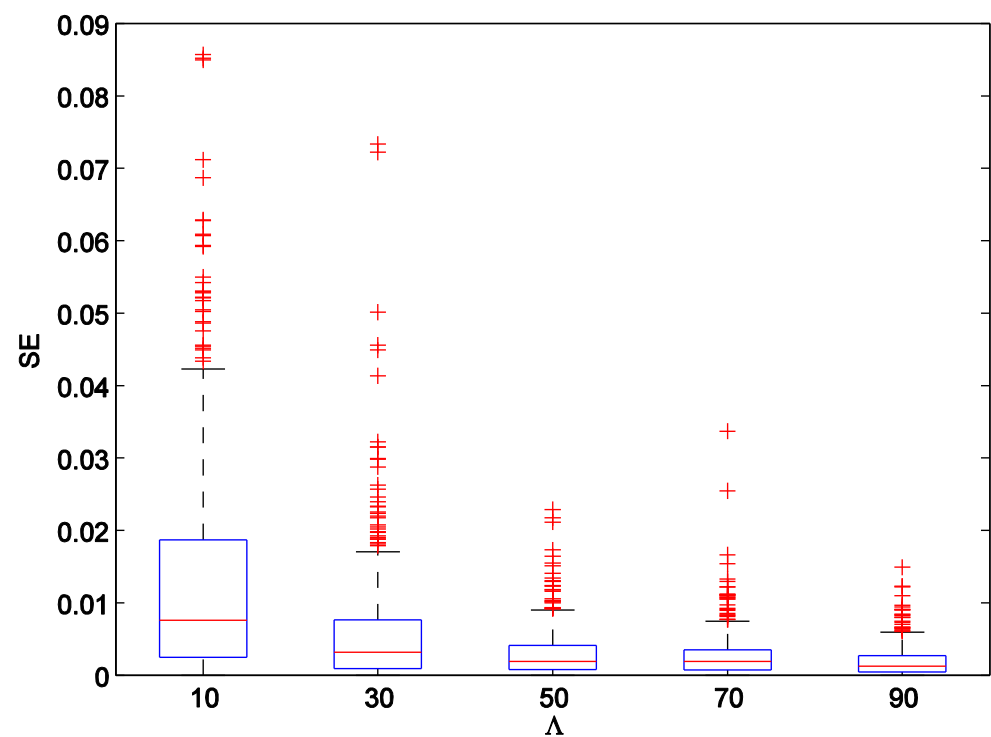

Figure 4.8: SE versus $\Lambda, \sigma_{0}^{2}=0.8, K=100$, and $M=16$ : digital case. 


\subsection{Effect of Signal-to-Noise Ratio on the estimation performance}

The dependence of $S E$ on the value of $S N R$ is presented in Figures 4.9, 4.10, 4.11 and 4.12. Four experiments are run with 500 Monte Carlo simulations for each $S N R$ value. The first experiment is performed to demonstrate the effect of varying the value of the Signalto-Noise Ratio of the observation channel $S N R_{0}$ on the $S E$. Three values are considered here for $S N R_{0}=0 \mathrm{~dB}, 10 \mathrm{~dB}$, and $20 \mathrm{~dB}$. The number of sensors is fixed to 10 sensors, the average field intensity $\Lambda$ is set to 100 and the noise variance of the transmission channel is fixed at $\sigma_{c}^{2}=0.003$. The results of the analog case are illustrated in Figure 4.9. It could be seen that as $S N R_{0}$ increases, better performance is achieved.

Figure 4.10 shows the results of varying $S N R_{0}$ against $S E$ in the case of using digital transmission channel. The number of quantization levels $M$ are assumed to be 8 levels.

The Signal-to-Noise Ratio of the transmission channel $S N R_{c}$ is varied to investigate its effect on the $S E$. Three values are assumed for $S N R_{c}=10 \mathrm{~dB}, 20 \mathrm{~dB}$, and $30 \mathrm{~dB}$, and $S N R_{0}$ is fixed to $20 \mathrm{~dB}$. Figure 4.11 shows the results of the analog case, and Figure 4.12 is for the digital case.

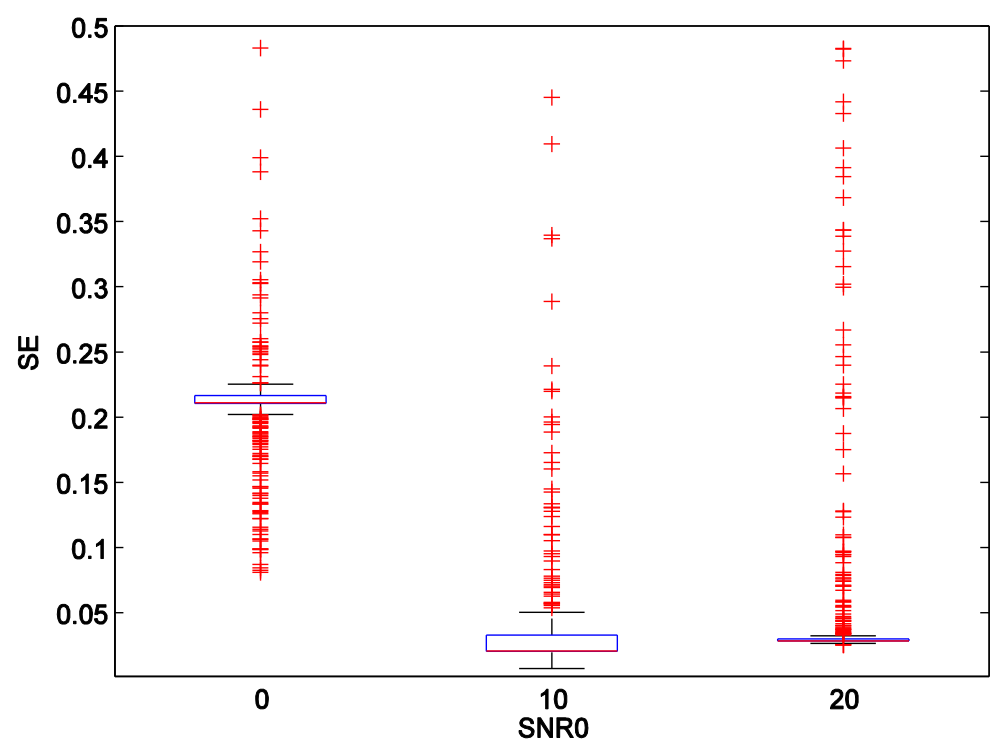

Figure 4.9: $\mathrm{SE}$ versus $S N R_{0}, \sigma_{c}^{2}=0.003, K=10$, analog case. 


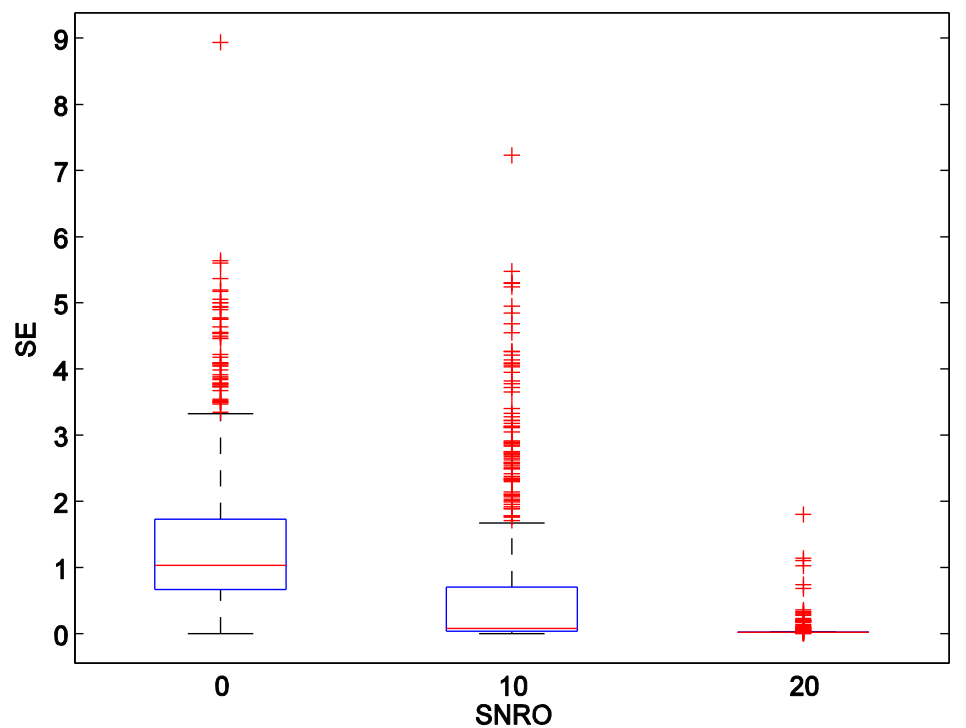

Figure 4.10: $\mathrm{SE}$ versus $S N R_{0}, S N R_{c}=40 \mathrm{~dB}, M=8, K=10$, digital case.

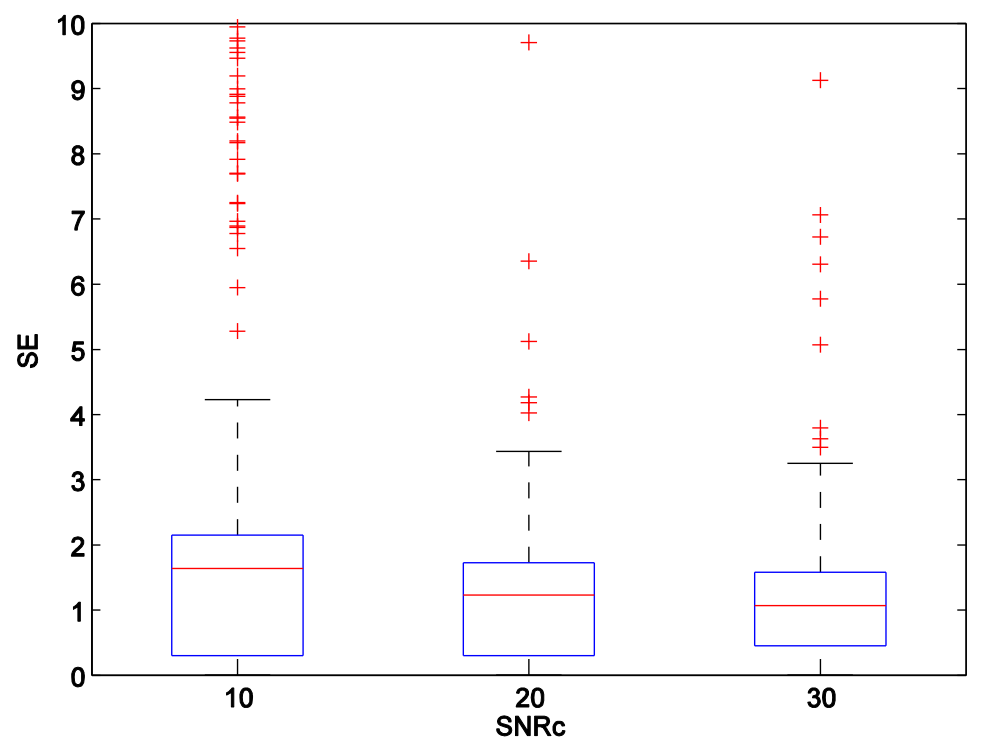

Figure 4.11: $\mathrm{SE}$ versus $S N R_{c}, S N R_{0}=20 \mathrm{~dB}, K=10$, analog case. 


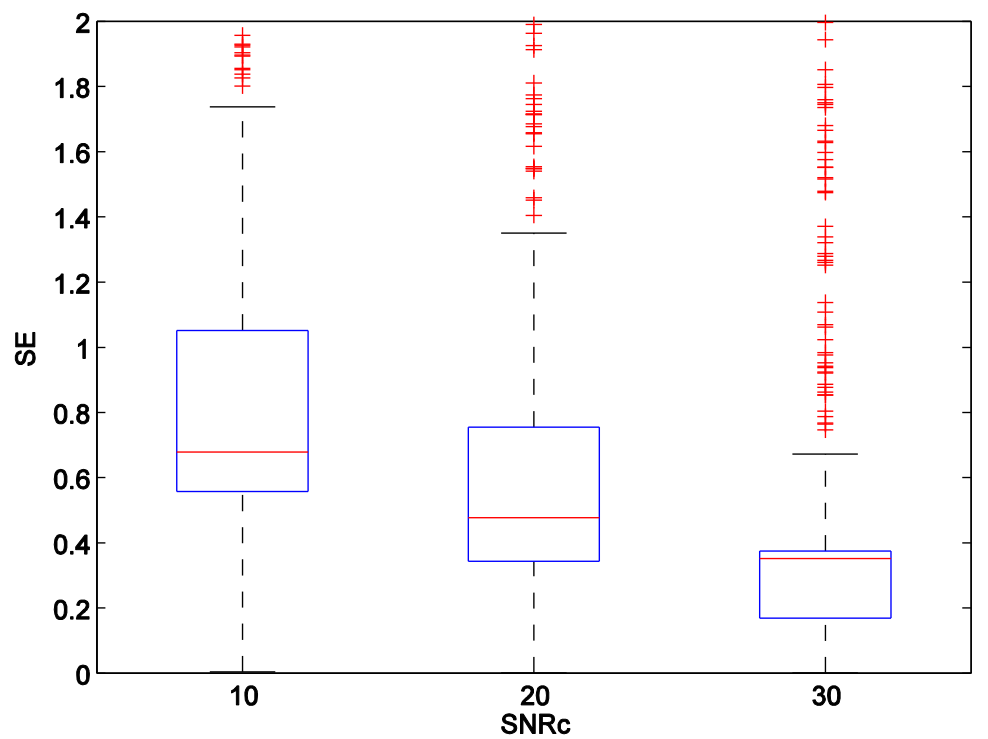

Figure 4.12: $\mathrm{SE}$ versus $S N R_{c}, S N R_{0}=20 \mathrm{~dB}, M=8, K=10$, digital case.

\subsection{Probability of Outliers}

We will now define another performance measure, the probability of outliers as

$$
\phi(\mu)=\operatorname{Pr}[S E>\mu]
$$

where $\mu$ is a positive threshold, and $\operatorname{Pr}$ stands for probability. Figures 4.13 and 4.14 present the probability of outliers as a function of the threshold $\mu$. The figures are generated using 1000 Monte Carlo simulations (in the analog case and digital cases). The number of sensors $K$ is set to 20 for the both cases. 


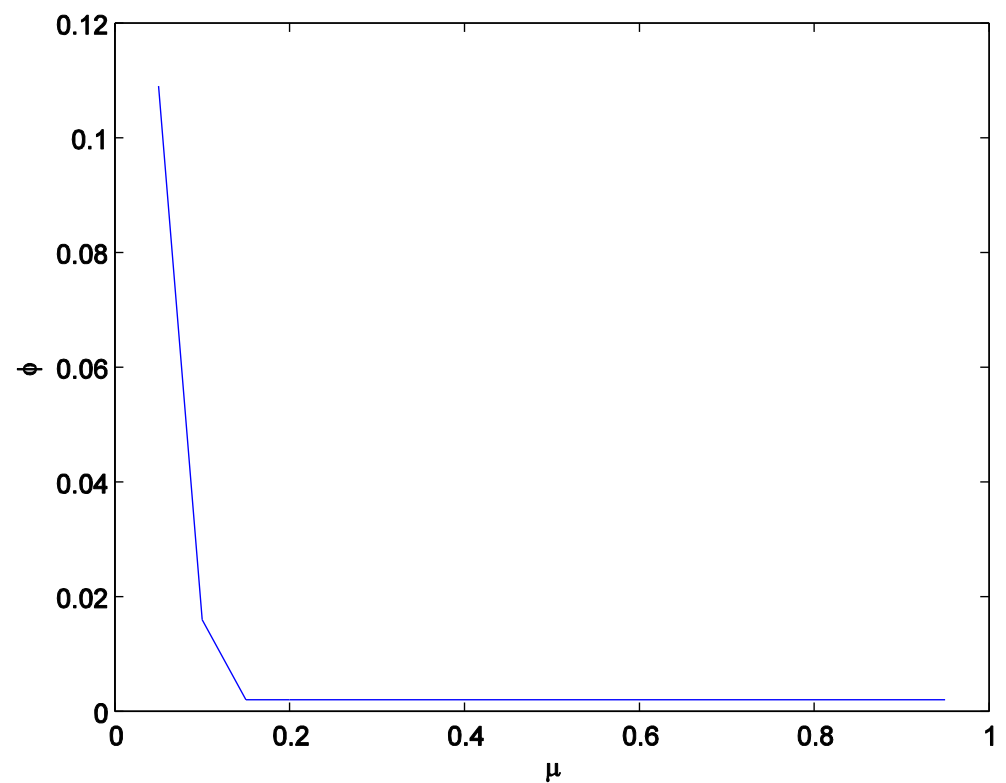

Figure 4.13: Probability of outliers, $\Lambda=100, K=20$, and $\sigma_{0}^{2}=0.8$ : analog case.

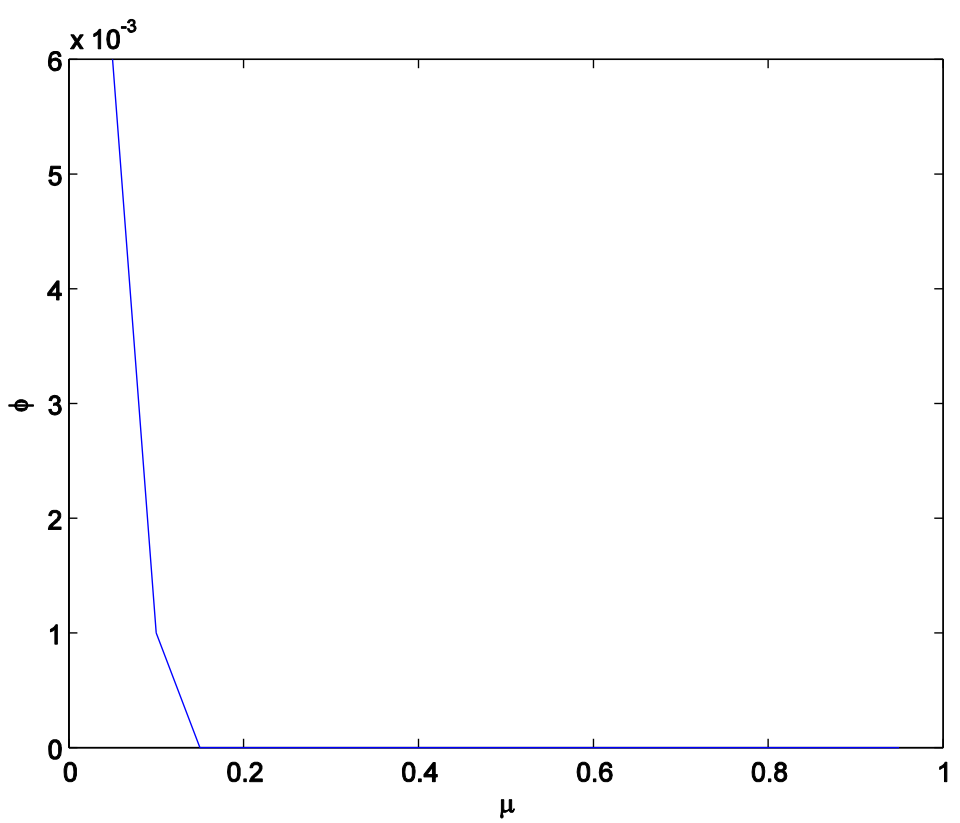

Figure 4.14: Probability of outlier, $\Lambda=100, K=20, \sigma_{0}^{2}=0.8$, and $M=16$ : digital case.

\subsection{Tracking Simulation}

Tracking simulations have been performed for both analog and digital cases. A true path is plotted together with the estimated path on the same plot. Since the algorithm requires 
two guessing points during initialization, one of them is selected as the previous location of the target modified by adding a small arbitrary value, and the other guessing point is the previous location plus another arbitrary value. The arbitrary value could be chosen as the radius of the labeled ring that meets a given $S E$ in the previous box plot graphs. This idea is similar to KALMAN linear prediction filter approach [55]. The first two guessing points are considered to be $(1,3)$ and $(3,1)$, as they are the boundaries of the area, and any $X$ or $Y$ point is bracketed in this range. In addition, there is another way to assume that the first true location is known with some arbitrary error, and the first two guessing points could be simulated as the first true target location augmented by random error, which could be taken in account by adding rand MATLAB function to the position in the MATLAB code.

The following graphs present tracking simulations with 100 sensors, $\Lambda=100$, and $\sigma_{0}^{2}=\sigma_{c}^{2}$ $=0.8$. By substituting these values in equations (4.1), (4.6), and (4.8), we have $S N R_{0}=27$ $d B, S N R_{c}=26 d B$ in the digital channel case, and $46 d B$ in the case of analog channel. These are the best $S N R$ values, as they are calculated by assuming the target is located at the area center, while the worst $S N R$ could be determined by supposing the target is located at one of corners of the square area.

Figure 4.15 illustrates a true path drawn as an exponentially decaying curve. The true target location is denoted by $(X C R, Y C R)$, and the position of the estimated target is located at 7 locations. The first point is assumed to be known with random error, the error is Gaussian distributed between 0 and 1, and the used communication technique is analog.

Figure 4.16 presents a true path curve shaped as $V$ with 11 estimated points. This is the case of digital communication with 16 quantization levels. 


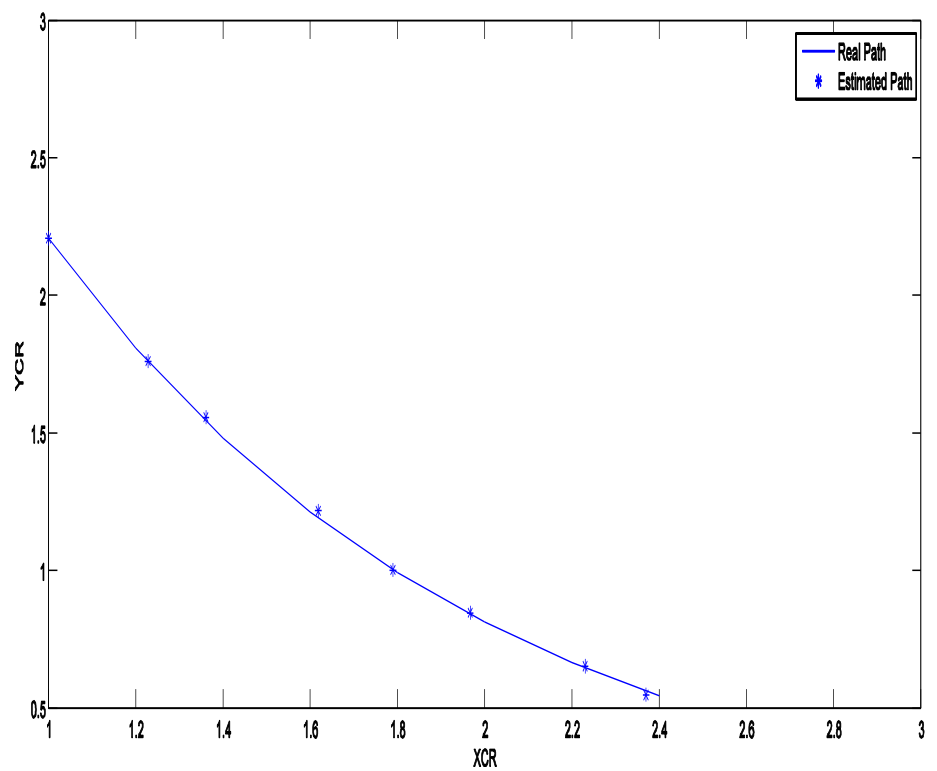

Figure 4.15: Tracking Simulation, $\Lambda=100, S N R_{0}=27 d B, \sigma_{c}^{2}=0.8$, analog case.

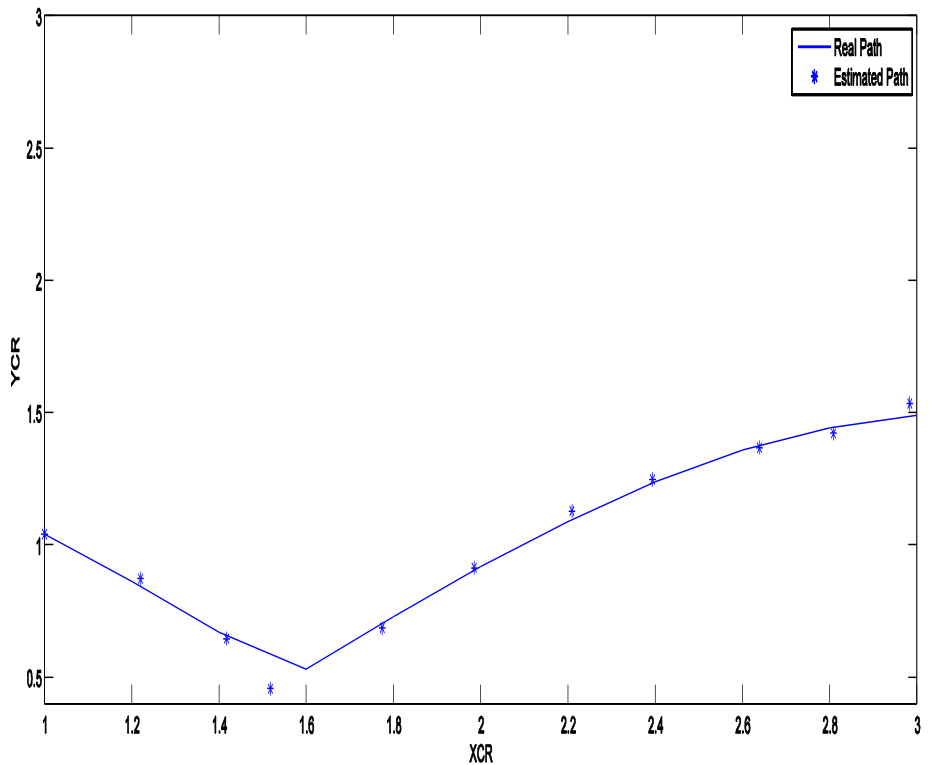

Figure 4.16: Tracking Simulation, $\Lambda=100, S N R_{0}=27 d B, \sigma_{c}^{2}=0.8, M=16$, digital case.

Figure 4.17 displays the same $V$-curve path. The estimates are obtained using analog communication. Figure 4.18 is for digital case with exponential path. 


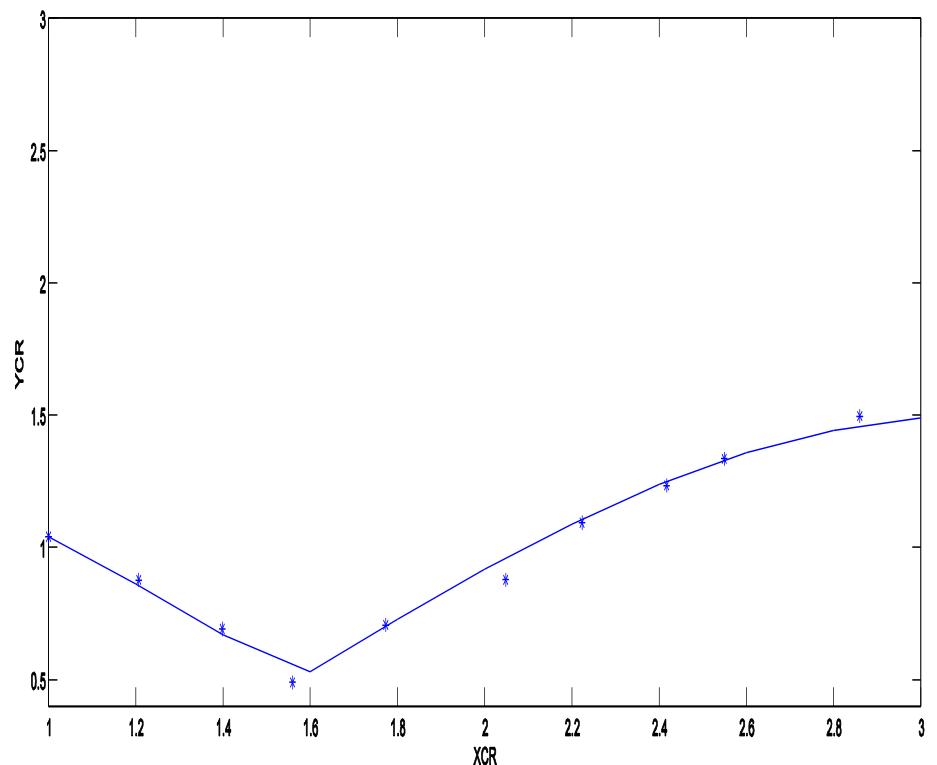

Figure 4.17: Tracking Simulation, $\Lambda=100, S N R_{0}=27 d B, \sigma_{c}^{2}=0.8$, analog case.

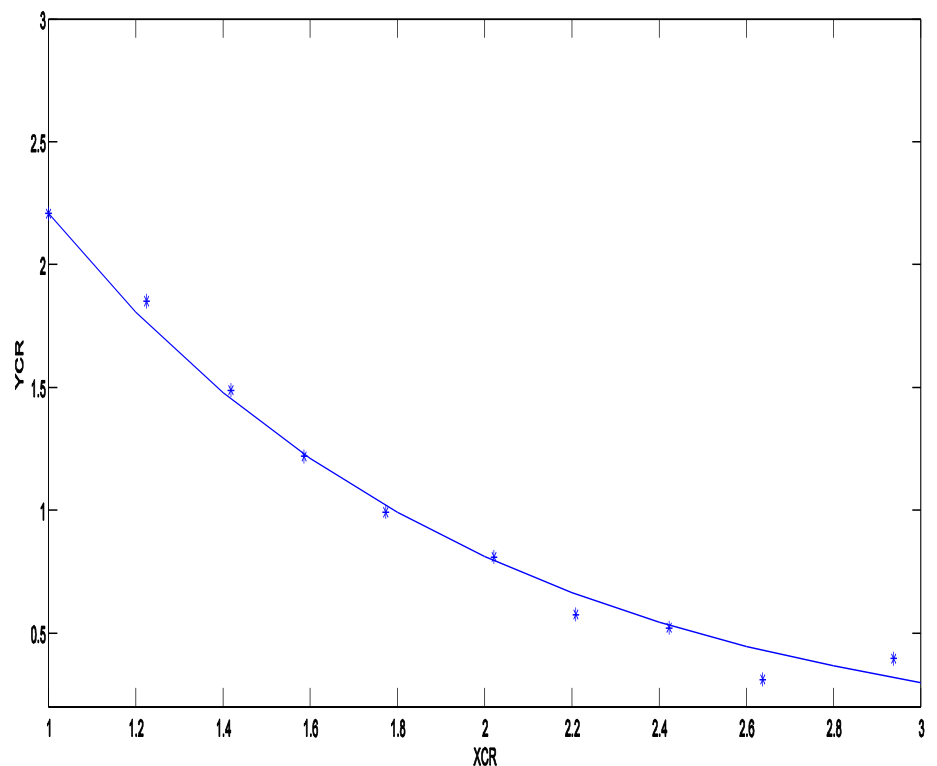

Figure 4.18: Tracking Simulation, $\Lambda=100, S N R_{0}=27 d B, \sigma_{c}^{2}=0.8, M=16$, digital case. 
Figure 4.19 shows a zig zag path. Data are transmitted over analog communication channel.

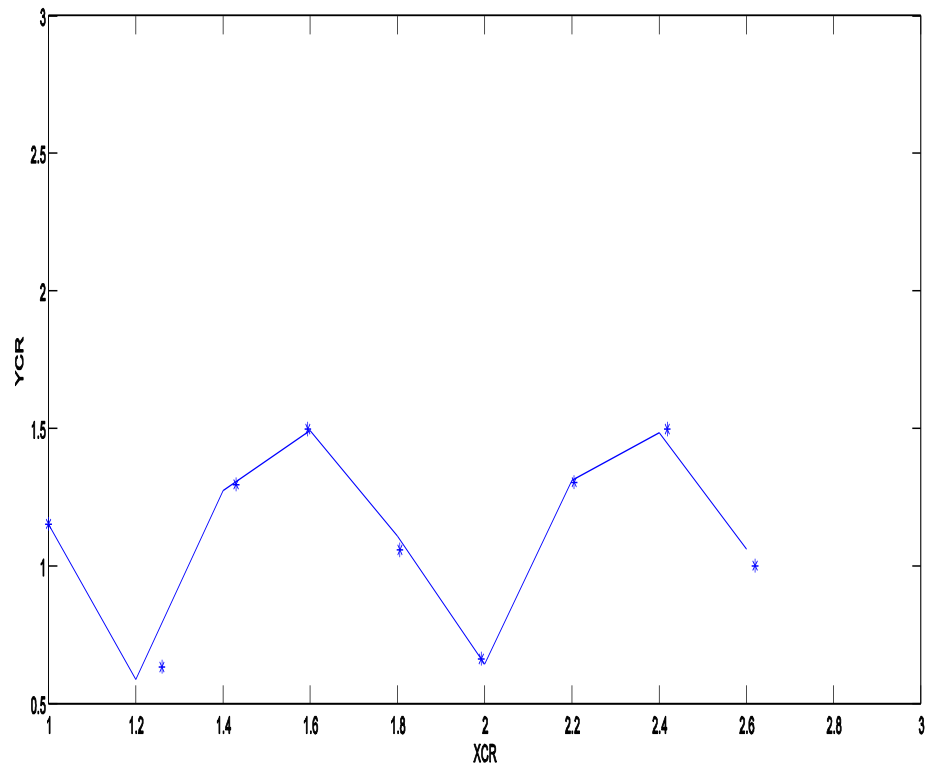

Figure 4.19: Tracking Simulation, $\Lambda=100, S N R_{0}=27 d B, \sigma_{c}^{2}=0.8$, analog case.

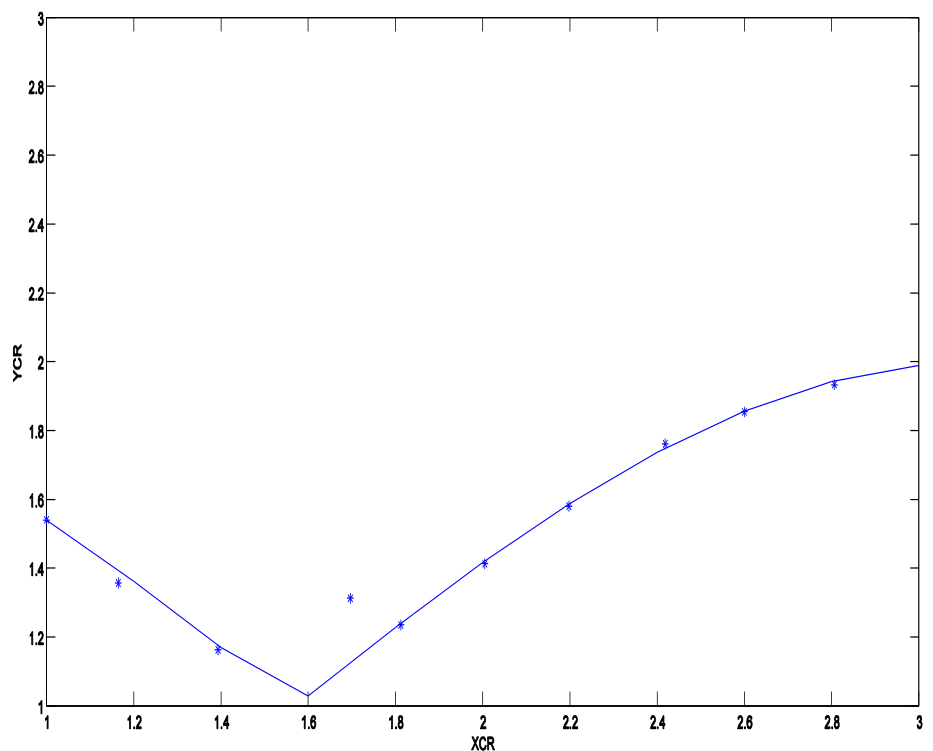

Figure 4.20: Tracking Simulation, $\Lambda=100, K=400, S N R_{0}=27 d B, \sigma_{c}^{2}=0.8$, analog case. 
Figure 4.20 presents tracking simulation with more sensors, $K=400$. By comparing it with Figure 4.17, we can note the increasing accuracy of the location estimation.

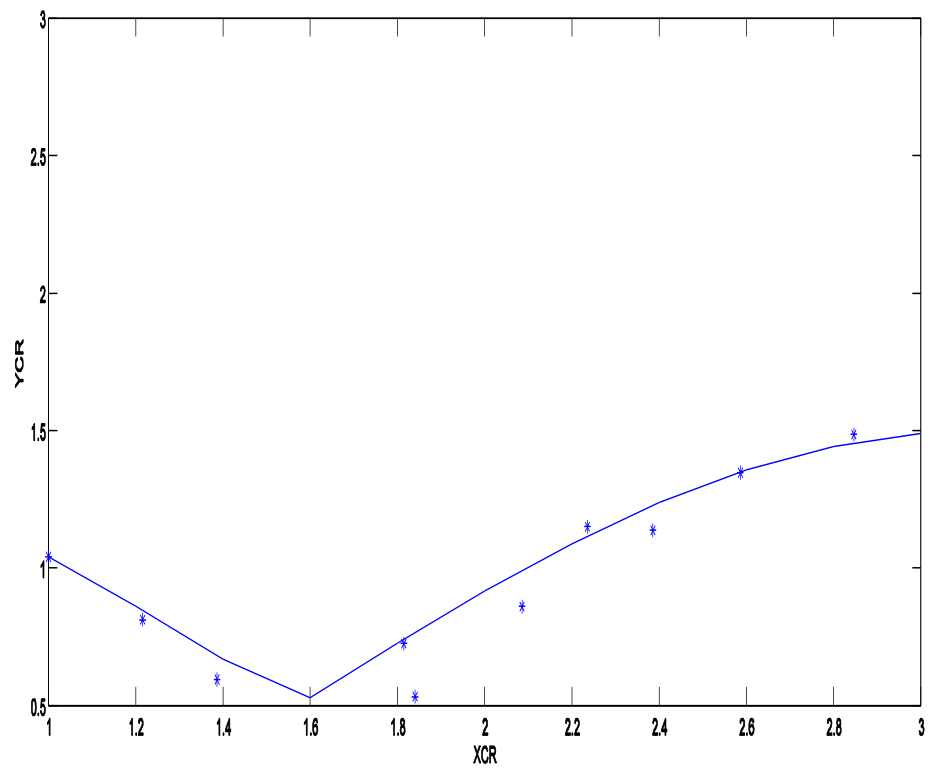

Figure 4.21: Tracking Simulation, $\Lambda=100, K=100, S N R_{0}=27 d B, \sigma_{c}^{2}=0.8, M=8$, digital case.

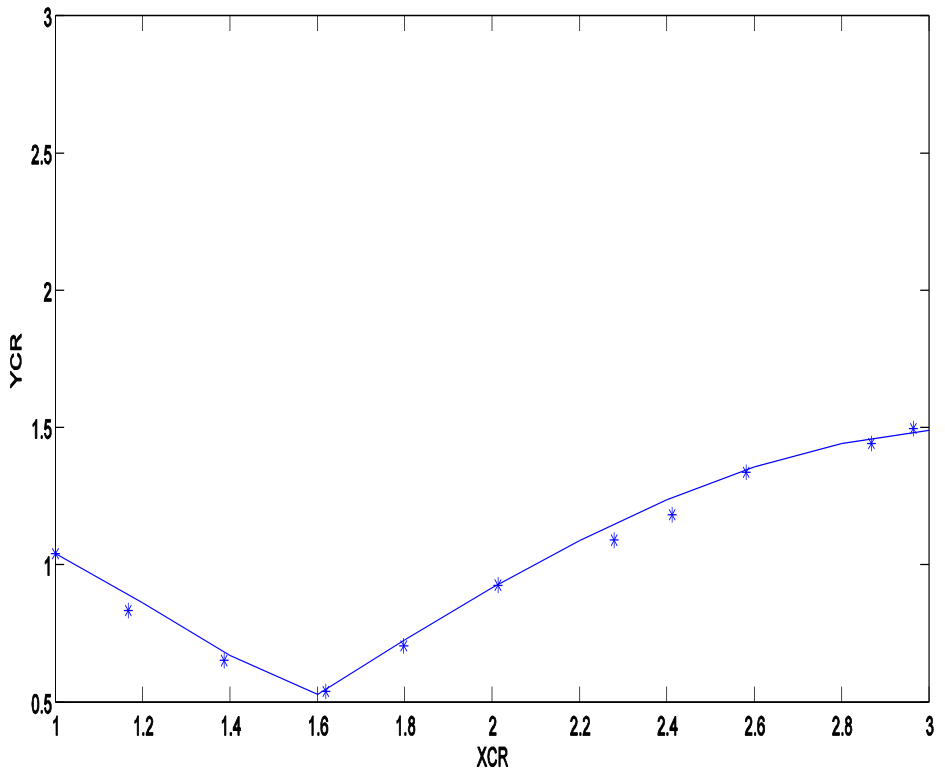

Figure 4.22: Tracking Simulation, $\Lambda=100, K=100, S N R_{0}=27 d B, \sigma_{c}^{2}=0.8, M=32$, digital case. 
Figures 4.21 and 4.22 illustrate the difference in location estimation accuracy between 8level and 32-level in the digital case. With more quantization steps, the median $S E$ reduces from 0.0096 (in the case of 8-levels) to 0.0018 (in the case of 32-levels).

All the previous graphs are generated with a single Monte Carlo realization for each location, and by assuming relatively high Signal-to-Ratio in the observation channel. In order to test the algorithm robustness under lower $S N R$ value, Figures 4.23 and 4.24 are produced with 100 Monte Carlo simulations for each target position, and by setting $S N R_{0}$ to $15 d B$, and $\Lambda=30$.

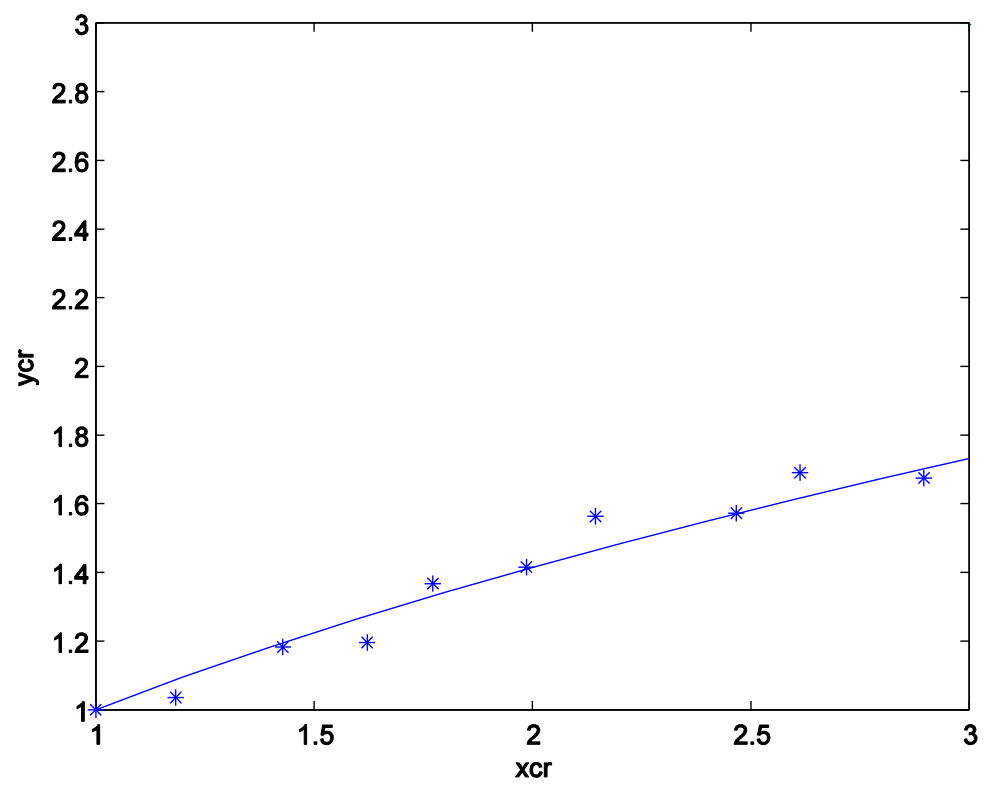

Figure 4.23: Tracking Simulation, $\Lambda=30, K=100, S N R_{0}=15 d B, \sigma_{c}^{2}=0.8$, analog case. 


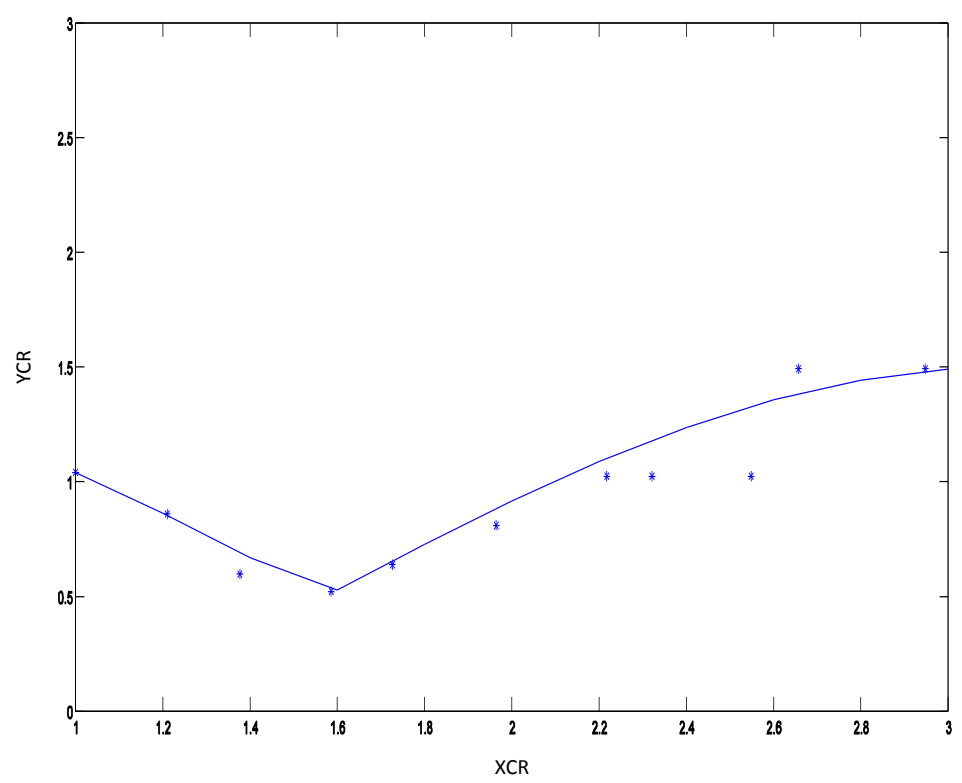

Figure 4.24: Tracking Simulation, $\Lambda=30, K=100, S N R_{0}=15 d B, \sigma_{c}^{2}=0.8, M=32$, digital case.

For the following experiments, we choose to initialize our iterative algorithm with a pair of extreme points $(1,1)$ and $(3,3)$. The points are substituted in the equations $(3.13),(3.14)$, (3.24) and (3.25), to check if the results have opposite signs; if not, the points are shifted by 0.001 till the opposite signs are achieved. Therefore, the proposed algorithm in Chapter 3 is run to estimate the target location. The initialization points here are the most remote points from the true location, so they are considered as the worst case in terms of $S E$ values. In addition, the first location is assumed to be unknown, unlike the preceding method. Figures 4.25 and 4.26 present the results of the simulations. 


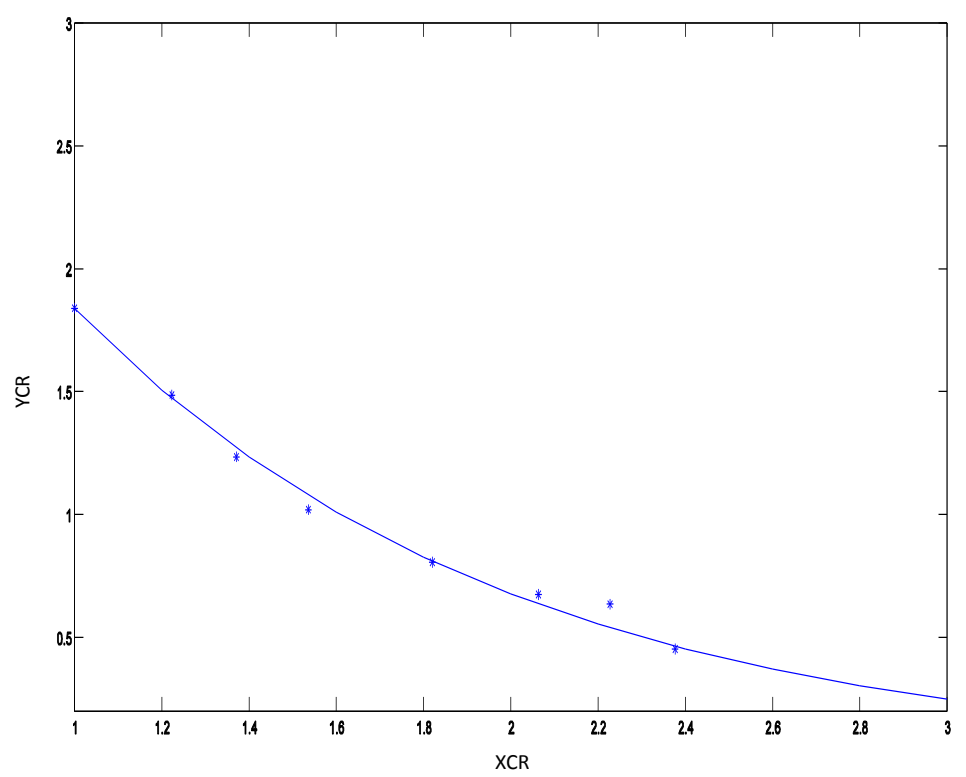

Figure 4.25: Tracking Simulation at a pair of points on the boundary of the area: $\Lambda=100$, $K=100, S N R_{0}=27 d B, \sigma_{c}^{2}=0.8$, analog case.

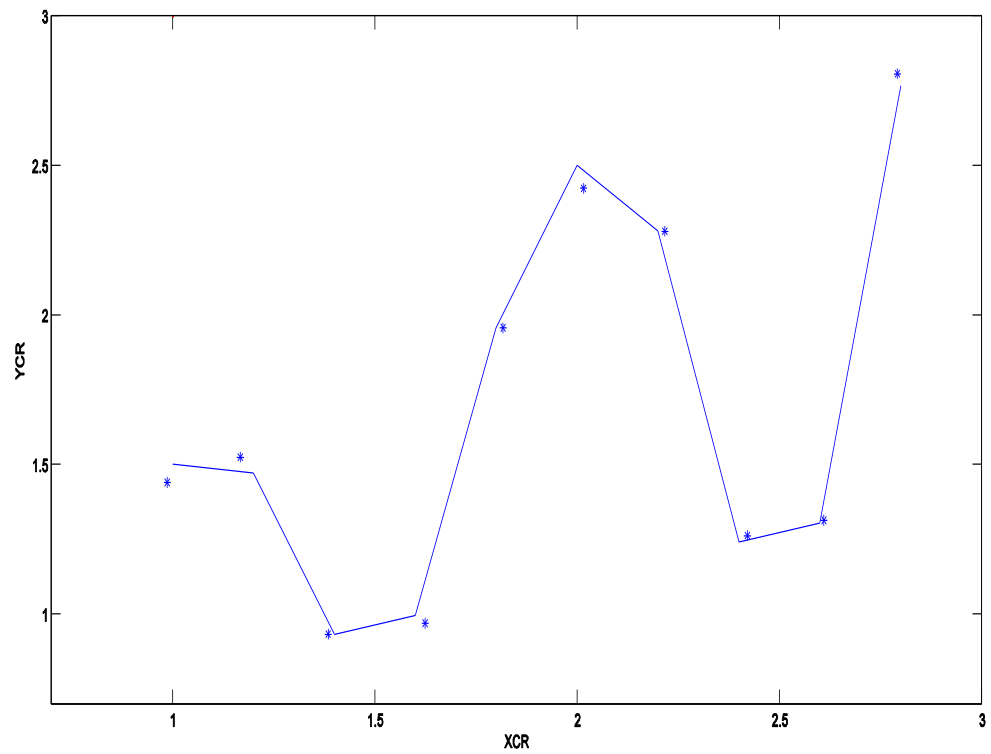

Figure 4.26: Tracking Simulation with initializations at the boundary of the area: $\Lambda=100$, $K=100, S N R_{0}=27 d B, \sigma_{c}^{2}=0.8$, digital case. 


\section{Chapter 5}

\section{Conclusion, discussion, and future work}

\subsection{Summary}

In this thesis, we proposed ML solution to the problem of distributed estimation of parametric stochastic field generated by a radiological point target. The purpose is to estimate the location of the target $\left(x_{c}, y_{c}\right)$. The solution is implemented in the form of a robust iterative algorithm.

The network model assumes a Gaussian receiver noise added to the target field, which acts as an inhomogeneous Poisson process. The sensor output is added to the noise of the receiver at the Fusion Center.

In the analog case, the model considers sensory data sent immediately over the channel to the FC. The joint $p d f$ of measurements at the FC is evaluated. Generally, the iterative solution for the analog case converges with smaller estimation error for the same number of sensors compared to the digital case, because the sensor measurements (samples) do not experience any quantization error. The digital case model includes extra processing step before the sensory data are sent over the channel. They are quantized by $M$ levels quantizer.

Bisection and Secant methods are jointly utilized to solve for ML estimates of unknown parameters $\left(x_{c}, y_{c}\right)$. Starting with two initial points (with a guarantee that the location is included between the initial points) facilitates finding the true location, because it is 
bracketed within known points. By using this algorithm the target can be localized with a small estimation error as presented in Chapter 4. The algorithm can also follow the target using a relatively low number of sensors and only few quantization levels (in the digital case). As shown in Figures 4.3 and 4.7, the digital model works better under low target intensity field $\Lambda$, while the analog channel performs better in the case of low sensors number as illustrated in Figures 4.1 and 4.4 .

Figures 4.2, 4.6, 4.25, and 4.26 show that the algorithm can be carried out with an acceptable performance, in the case of high initial point deviation. The initial values are set to the area boundaries in Figures 4.25 and 4.26.

The joint pdf of measurements at the FC for the digital case is derived. It is more complex compared to the function in the analog case. Therefore more iterations are required for convergence, and this requires faster FC processor than the analog case. On the other hand, the existence of the common quantizer in the digital case gives the option of adjusting the accuracy, signal power, and the required channel bandwidth.

\subsection{Claimed Novelties}

The following novelties are claimed in this thesis:

1. Tracking a target generating a random field, the field is modeled as spatial Poisson process.

2. A compound solution combining Bisection and Secant methods is applied to solve for ML estimates of unknown parameters. The solution is fast and exhibits good convergence.

3. Numerical analysis is performed. Both digital and analog scenarios are analyzed. SE is analyzed as a function of sensors in WSN, $S N R_{o}, S N R_{c}$, and quantization levels. Tracking examples are generated. 


\subsection{Future Work}

This work can be extended to many ways. Here are only a few examples:

- Instead of only one target, the work could be extended to localize multi-target objects.

- This work assumes a network of the same type sensors (homogeneous network), and could be extended to include heterogeneous sensors.

- Different modulation methods could be adopted to test the effect of each method on the system performance.

- The used quantizer here is common for all the sensors, assigning a dynamic quantizer for each sensor is a worthy subject for further research study.

- The sensors in our thesis are assumed to be ideal, another research could be issued by supposing some malfunctioning sensors in the network (byzantine attack).

- More sources of distortions can be considered in the channel, such as fading, multi-path loss, and interference. 


\section{References}

[1] N. Sharma, M. K. Singh, and A. Goswami, "A heuristic approach for component selection of low power micro sensor nodes based on simple energy model," International Conference on Electronics Computer Technology, vol. 4, pp. 290-295, April 2011.

[2] V. Raghunathan, C. Schurgers, S. Park, and M. B. Srivastava, "Energy-aware wireless microsensor networks," IEEE Signal Processing Magazine, vol. 19, no. 2, pp. 40 -50, March 2002.

[3] J. Xu, H. Wang, F. Lang, P. Wang, and Z. Hou, "Study on wsn topology division and lifetime," IEEE International Conference on Computer Science and Automation Engineering, vol. 1, pp. 380 -384, June 2011.

[4] C. S. Hsu, J. P. Sheu, and Y. J. Chang, "Efficient broadcasting protocols for regular wireless sensor networks," International Conference on Parallel Processing, pp. $213-220$, October 2003.

[5] A. Shrestha and L. Xing, "A performance comparison of different topologies for wireless sensor networks," IEEE Conference on Technologies for Homeland Security, pp. 280 -285, May 2007.

[6] D. Enjie, H. Yanqiu, H. He, Y. Wanli, and L. Xuerui, "A linear-hierarchy wsn topology used in coal face," International Conference on New Trends in Information Science and Service Science, pp. 159 -163, May 2010.

[7] G. Thatte and U. Mitra, "Power allocation in linear and tree wsn topologies," Fortieth Asilomar Conference on Signals, Systems and Computers, pp. $1342-1346$, November 2006.

[8] S. Giannoulis, A. Prayati, C. Antonopoulos, and G. Papadopoulos, "A.mo.r : An adaptive routing mechanism for wsn health applications," International Conference on Pervasive Computing Technologies for Healthcare, pp. 1-4, April 2009.

[9] D. Baghyalakshmi, J. Ebenezer, and S. A. V. SatyaMurty, "Wireless sensor network based temperature monitoring for high performance computing cluster," International Conference on Recent Trends in Information Technology, pp. 1105 -1110 , June 2011.

[10] W. Wang and S. Cao, "Application research on remote intelligent monitoring system of greenhouse based on zigbee wsn," International Congress on Image and Signal Processing, pp. 1 -5, October 2009. 
[11] E. H. Sim and L. L. Yang, "Ds-cdma with m-ary orthogonal modulation for wireless sensor networks simultaneously monitoring multiple events," IEEE Vehicular Technology Conference, pp. 1 -5, May 2012.

[12] K. I. Wong, N. Barsoum, and C. Z. Myint, "Teaching the electronic design and embedded system course with body sensor nodes," International Conference on Education Technology and Computer, vol. 3, pp. 158 -162, June 2010.

[13] R. Machado and S. Tekinay, "Neural network-based approach for adaptive density control and reliability in wireless sensor networks," IEEE Wireless Communications and Networking Conference, pp. 2537 -2542, April 2008.

[14] D. G. Melese, H. Xiong, and Q. Gao, "Consumed energy as a factor for cluster head selection in wireless sensor networks," International Conference on Wireless Communications Networking and Mobile Computing, pp. 1 -4, September 2010.

[15] T. Wu and Q. Cheng, "Distributed estimation over fading channels using one-bit quantization," Asilomar Conference on Signals, Systems and Computers, pp. 1968 -1972, October 2008.

[16] M. Chen, V. C. Leung, S. Mao, and M. Li, "Cross-layer and path priority scheduling based real-time video communications over wireless sensor networks," IEEE Vehicular Technology Conference, pp. 2873 -2877, May 2008.

[17] W. Gao, H. Zhao, C. Song, and J. Xu, "A new distributed particle filtering for wsn target tracking," International Conference on Signal Processing Systems, pp. $334-337$.

[18] M.Z. Rahman, D. Habibi, and I. Ahmad, "Source localisation in wireless sensor networks based on optimised maximum likelihood," Telecommunication Networks and Applications Conference, pp. 235 -239, December 2008.

[19] T. V. Waterschoot and G. Leus, "Static field estimation using a wireless sensor network based on the finite element method," IEEE International Workshop on Computational Advances in Multi-Sensor Adaptive Processing, pp. 369 -372, December 2011.

[20] J. C. P. Bus and T. J. Dekker, "Two efficient algorithms with guaranteed convergence for finding a zero of a function," ACM Transactions on Mathematical Software, vol. 1, no. 4, pp. 330-345, December 1975.

[21] B. A Myron and E. L. Isaacson, Numerical Analysis for Applied Science, Wiley, 1997.

[22] R. Brent, Algorithms for Minimization without Derivatives, Prentice-Hall, Englewood Cliffs, NJ, 1973.

[23] A. Ribeiro and G. B. Giannakis, "Bandwidth-constrained distributed estimation for wireless sensor networks-part 1: Gaussian case," IEEE Transactions on Signal Processing, vol. 54, no. 3, pp. 1131 - 1143, March 2006.

[24] J. Li and G. AlRegib, "Distributed estimation in energy-constrained wireless sensor networks," IEEE Transactions on Signal Processing, vol. 57, no. 10, pp. 3746 -3758, October 2009. 
[25] J. J. Xiao and Z. Q. Luo, "Decentralized estimation in an inhomogeneous sensing environment," IEEE Transactions on Information Theory, vol. 51, no. 10, pp. $3564-3575$, October 2005.

[26] R. Al Alawi, "Rssi based location estimation in wireless sensors networks," IEEE International Conference on Networks, pp. 118 -122, December 2011.

[27] C. Hongyang, D. Ping, X. Yongjun, and L. Xiaowei, "A robust location algorithm with biased extended kalman filtering of tdoa data for wireless sensor networks," International Conference on Wireless Communications, Networking and Mobile Computing, vol. 2, pp. 883 -886, September 2005.

[28] O. Ozdemir, R. Niu, and P. K Varshney, "Channel aware target localization with quantized data in wireless sensor networks," IEEE Transactions on Signal Processing, vol. 57, no. 3, pp. 1190 -1202, March 2009.

[29] K. Agrawal, A. Vempaty, H. Chen, and P. K. Varshney, "Target localization in wireless sensor networks with quantized data in the presence of byzantine attacks," Conference on Signals, Systems and Computers, pp. 1669 -1673, November 2011.

[30] R. Niu and P. K. Varshney, "Target location estimation in sensor networks with quantized data," IEEE Transactions on Signal Processing, vol. 54, no. 12, pp. 4519 -4528, December 2006.

[31] J. Jia, M. Liu, and X. Li, "Acoustic passive localization algorithm based on wireless sensor networks," International Conference on Mechatronics and Automation, pp. 1145 -1149, August 2009.

[32] S. Wang, X. Wang, Y. Wang, and X. Sun, "Distributed lightweight target tracking for wireless sensor networks," IEEE International Conference on Mobile Adhoc and Sensor Systems, pp. 870 -875, October 2009.

[33] J. Wei and X. Zhang, "Decentralized-detection based mobile multi-target tracking in wireless sensor networks," IEEE International Conference on Communications, pp. $1-5$, May 2010.

[34] R. Sangeetha and B. Kalpana, "Denoising the signals using kalman filter for target tracking in wireless sensor networks," International Conference on Electronics Computer Technology, vol. 2, pp. 254-258, April 2011.

[35] A. A. Kumar and K. M. Sivalingam, "Target tracking in a wsn with directional sensors using electronic beam steering," International Conference on Communication Systems and Networks, pp. 1 -10, January 2012.

[36] M. Mansouri, L. Khoukhi, H. Nounou, and M. Nounou, "Genetic algorithm optimization for quantized target tracking in wireless sensor networks," IEEE Global Telecommunications Conference, pp. 1 -6, December 2011.

[37] K. Sudakshina, Analog and Digital Communications, Pearson Education India, New Delhi, 2010.

[38] L. Hanzo, P. Cherriman, and J. Streit, Wireless Video Communications: Second to Third Generation and Beyond, Wiley-IEEE Press, 2001. 
[39] D. L. Snyder and M. I. Michael, Random point processes in time and space, Springer-Verlag, New York, 1991.

[40] Elliott. R, Electromagnetics: History, Theory, and Applications, Wiley-IEEE press ebook chapters, 1993.

[41] X. Gong, S. A Vorobyov, and C. Tellambura, "Optimal bandwidth and power allocation for sum ergodic capacity under fading channels in cognitive radio networks," IEEE Transactions on Signal Processing, vol. 59, no. 4, pp. 1814-1826, April 2011.

[42] A. Gersho and R. M. Gray, Vector Quantization and Signal Compression, Springer, New York, 1991.

[43] W. H. Press, S. Teukolsky, B. P. Flannery, and W. T. Vetterling, Numerical Recipes: The Art of Scientific Computing (3rd ed), Cambridge University Press, New York, 2007.

[44] H. V. Poor, An introduction to signal detection and estimation, Springer, New York, 1994.

[45] B. Eisenberg and R. Sullivan, "Why is the sum of independent normal random variables normal?," Mathematical Association of America, Mathematics Magazine, vol. 81, no. 5, pp. 362-366, December 2008.

[46] M. C Vassilis and P. N Timothy, Elements of Mathematics for Economics and Finance, Springer, London, 2007.

[47] S. Miller and D. Childers, Probability and random processes, Elsevier Inc, 2012.

[48] W. Li, Risk Assessment Of Power Systems: Models, Methods, and Applications, Wiley-IEEE Press, 2005.

[49] E. Suli and D. Mayers, An introduction to numerical analysis, Cambridge University Press, 2003.

[50] R. L. Burden and J. D. Faires, Numerical analysis, Boston-PWS Pub, 1993.

[51] M. B. Allen and E. L. Isaacson, Numerical Analysis for Applied Science, Wiley, 1997.

[52] B. Guerin and G. El Fakhri, "Realistic pet monte carlo simulation with pixelated block detectors, light sharing, random coincidences and dead-time modeling," IEEE Transactions on Nuclear Science, vol. 55, no. 3, pp. 942 -952, June 2008.

[53] S Wolfram, "Wolfram alpha, polar integration calculator, feb 2 2013, http://www.wolframalpha.com," .

[54] A. Garcia, Probability and Random Process for Electrical Engineering, AddisonWesley Publishing Company, Inc, 1994.

[55] H. Ruskeepaa, "A quasi-linear estimation method-application to kalman filtering with stochastic regressors," IEEE Transactions on Automatic Control, vol. 30, no. 8, pp. $767-771$, August 1985. 